\title{
An experimental and kinetic modeling study of cyclohexane pyrolysis at low pressure
}

\author{
Zhandong Wang a,b ${ }^{\mathrm{a}}$ Zhanjun Cheng ${ }^{\mathrm{a}, \mathrm{b}}$, Wenhao Yuan ${ }^{\mathrm{a}, \mathrm{b}}$, Jianghuai Cai ${ }^{\mathrm{a}, \mathrm{b}}$, Lidong Zhang ${ }^{\mathrm{b}}$, \\ Feng Zhang ${ }^{b}$, Fei $\mathrm{Qi}^{\mathrm{a}, \mathrm{b}, *}$, Jing Wang ${ }^{\mathrm{c}}$ \\ ${ }^{a}$ State Key Laboratory of Fire Science, University of Science and Technology of China, Hefei, Anhui 230026, PR China \\ ${ }^{\mathrm{b}}$ National Synchrotron Radiation Laboratory, University of Science and Technology of China, Hefei, Anhui 230029, PR China \\ ' Institute of Mechanics, Chinese Academy of Sciences, Beijing 100190, PR China
}

\section{A R T I C L E I N F O}

\section{Article history:}

Received 26 October 2011

Received in revised form 13 February 2012

Accepted 19 February 2012

Available online 16 March 2012

\section{Keywords:}

Cyclohexane pyrolysis

Synchrotron VUV photoionization mass

spectrometry

Kinetic modeling

1-Hexene

Benzene formation

\begin{abstract}
A B S T R A C T
The pyrolysis of cyclohexane at low pressure (40 mbar) was studied in a plug flow reactor from 950 to $1520 \mathrm{~K}$ by synchrotron VUV photoionization mass spectrometry. More than 30 species were identified by measurement of photoionization efficiency (PIE) spectra, including some radicals like methyl, propargyl, allyl and cyclopentadienyl radicals, and stable products (e.g., 1-hexene, benzene and some aromatics). Among all the products, 1-hexene is formed at the lowest temperature, indicating that the isomerization of cyclohexane to 1-hexene is the dominant initial decomposition channel under the condition of our experiment. We built a kinetic model including 148 species and 557 reactions to simulate the experimental results. The model satisfactorily reproduced the mole fraction profiles of most pyrolysis products. The rate of production (ROP) analysis at 1360 and $1520 \mathrm{~K}$ shows that cyclohexane is consumed mainly through two reaction sequences: cyclohexane $\rightarrow 1$-hexene $\rightarrow$ allyl radical $+n$-propyl radical, and cyclohexane $\rightarrow$ cyclohexyl radical $\rightarrow$ hex-5-en-1-yl radical that further decomposes to 1,3-butadiene via hex-1-en-3-yl and but-3-en-1-yl radicals. Besides the stepwise dehydrogenation of cyclohexane, $\mathrm{C} 3+\mathrm{C} 3$ channels, i.e. $\mathrm{C}_{3} \mathrm{H}_{3}+\mathrm{C}_{3} \mathrm{H}_{3}$ and $\mathrm{C}_{3} \mathrm{H}_{3}+\mathrm{aC}_{3} \mathrm{H}_{5}$ also have important contribution to benzene formation. The simulation reveals that $\mathrm{C}_{3} \mathrm{H}_{3}+\mathrm{C}_{3} \mathrm{H}_{3}=$ phenyl $+\mathrm{H}$ reaction is the key step for other aromatics formation, i.e. toluene, phenylacetylene, styrene, ethylbenzene and indene in this work.
\end{abstract}

(c) 2012 The Combustion Institute. Published by Elsevier Inc. All rights reserved.

\section{Introduction}

Cycloalkanes widely exist in diesel, jet, and gasoline fuels. They make up to $35 \%$ of conventional diesel fuels, about $20 \%$ of jet fuels and about $10 \%$ of gasoline $[1,2]$. Among cycloalkanes, cyclohexane has received great attention due to its simplicity, and the role as a good starting point to develop cycloalkane models. Many experiments have been carried out to investigate pyrolysis [3-6], oxidation [7-17] and combustion [18-25] of cyclohexane. In addition to experimental investigations, some theoretical studies have been performed by Handford-Styring and Walker [26], Sirjean et al. [27] and Cavallotti et al. [28].

However, despite the great effort towards cyclohexane, some aspects of cyclohexane chemistry are still not fully understood. Tsang [29] and Brown et al. [30] proposed that the dominant initial channel of cyclohexane pyrolysis is its isomerization to form 1hexene. However, the experimental results in an annular reactor

* Corresponding author at: National Synchrotron Radiation Laboratory, University of Science and Technology of China, Hefei, Anhui 230029, PR China. Fax: +86 5515141078.

E-mail address: fqi@ustc.edu.cn (F. Qi). at atmospheric pressure obtained by Aribike et al. [31] did not confirm Tsang's prediction [29], since 1-hexene was not observed. Voisin et al. [8] performed a measurement for the oxidation of cyclohexane in a jet stirred reactor (JSR) at 10 atm, in which 1-hexene was also not identified. The JSR oxidation experiment carried out by EI Bakali et al. [9] at 1,2 and $10 \mathrm{~atm}$ showed that a pressure decrease favored the formation of olefins. In their experiments, 1hexene was observed at $1 \mathrm{~atm}$, but not at $10 \mathrm{~atm}$. Dissociation channels other than isomerization reaction of cyclohexane were proposed by Aribike et al. [31], Voisin et al. [8] and EI Bakali et al. [9] due to the absence of 1-hexene. Recently, Kiefer et al. [5] carried out shock tube and theoretical study of cyclohexane decomposition. Both experimental and theoretical results confirmed that cyclohexane predominantly isomerizes into 1-hexene.

Another aspect for cyclohexane chemistry is the mechanism of benzene formation. Besides recombination reactions of small linear hydrocarbons, there are many experimental evidences that benzene can be produced by dehydrogenation of cyclohexane $[8,10,13,18,32]$. In a sooting premixed flame, Ciajolo et al. [23] predicted that benzene comes from the fast dehydrogenation of cyclohexane at the preheat and reaction zones and from the recombination/addition reactions of small radicals, especially the 
self-combination of $\mathrm{C}_{3} \mathrm{H}_{3}$ at the postflame zone. The laminar flames of cyclohexane investigated by Law et al. [21] and Li et al. [25] showed that benzene is formed dominantly by stepwise dehydrogenation of cyclohexane under stoichiometric condition, and by the synergy of both fuel dehydrogenation and radical recombination under fuel-rich condition. However, McEnally and Pfefferle [20] showed that dehydrogenation is a minor pathway comparing with addition reactions for benzene formation in non-premixed cyclohexane flames.

In this work, we studied the cyclohexane pyrolysis at low pressure ( 40 mbar) in a plug flow reactor in the temperature range of 950-1520 K using synchrotron VUV photoionization mass spectrometry (SVUV-PIMS). This technique has been proven as a powerful in situ diagnostic method for complex reaction systems such as pyrolysis and combustion, low temperature oxidation and organic analysis [33-37]. The pyrolysis products, particularly 1-hexene and some aromatics were detected and quantified. A detailed kinetic model of cyclohexane pyrolysis was developed and validated by our experimental results. The main objective of this work is to discuss the initial decomposition channels of cyclohexane and the formation of benzene and larger aromatics under the conditions of this work.

\section{Experimental method}

The experimental work was carried out at U10 beamline of National Synchrotron Radiation Laboratory (NSRL) in Hefei, China. Description of the beamline and pyrolysis apparatus can be found in detail elsewhere $[38,39]$, and is briefly described here. The synchrotron radiation from a bend magnet beamline of the $800 \mathrm{MeV}$ electron storage ring is dispersed by a $1 \mathrm{~m}$ Seya-Namioka monochromator equipped with a 1200 grooves $/ \mathrm{mm}$ grating. The energy resolving power $(E / \Delta E)$ is about 500 at $\sim 150 \mu \mathrm{m}$ entrance and exit slits. A LiF window is mounted between the exit slit and the photoionization chamber to eliminate the higher-order harmonic radiation when the wavelength is longer than $105 \mathrm{~nm}$ (equal to $11.80 \mathrm{eV}$ ). A wavelength of $74.5 \mathrm{~nm}$ (equal to $16.65 \mathrm{eV}$ ) without LiF window was used to get the signals of $\mathrm{Ar}, \mathrm{H}_{2}$ and $\mathrm{CH}_{4}$, and measure the mixture of cold gases $\mathrm{Ar}, \mathrm{H}_{2}$ and $\mathrm{CH}_{4}$ for calibration of the major species.

The pyrolysis apparatus consists of a pyrolysis chamber containing a high temperature furnace, a differentially-pumped chamber with a molecular-beam sampling system, and a photoionization chamber combined with a home-made reflectron time-of-flight mass spectrometer (RTOF-MS). The pyrolysis species were sampled by a quartz cone with a $\sim 500 \mu \mathrm{m}$ orifice at the tip. The formed molecular beam passed through a nickel skimmer into the photoionization chamber, where it was crossed by the synchrotron light, and then the photoions were collected and analyzed by the RTOFMS.

A newly constructed furnace was used in this experiment. The furnace is similar to the former one that we used to study the pyrolysis of butene isomers [40], but the heating region is longer in this study. As shown in Fig. 1a, the heated region is $150 \mathrm{~mm}$ and the distance from the outlet of furnace to the sampling nozzle is about $10 \mathrm{~mm}$. A tungsten-rhenium thermocouple was put close to the middle region of the heating wire to monitor the outside temperature $\left(T_{\text {out }}\right)$ of the flow tube. Before performing the experiment, a K-type thermocouple was put inside the flow tube to measure the temperature profiles along the flow tube with a Ar flow rate of 1.0 standard liter per minute (SLM). The position of the K-type thermocouple can be controlled by a feedthrough outside the pyrolysis chamber, and then the temperatures at different positions along the flow tube axis can be measured, named $T_{\text {in }}(d)$. Here, $d$ refers to the position of the thermocouple. In this work, four $T_{\text {in }}(d)$

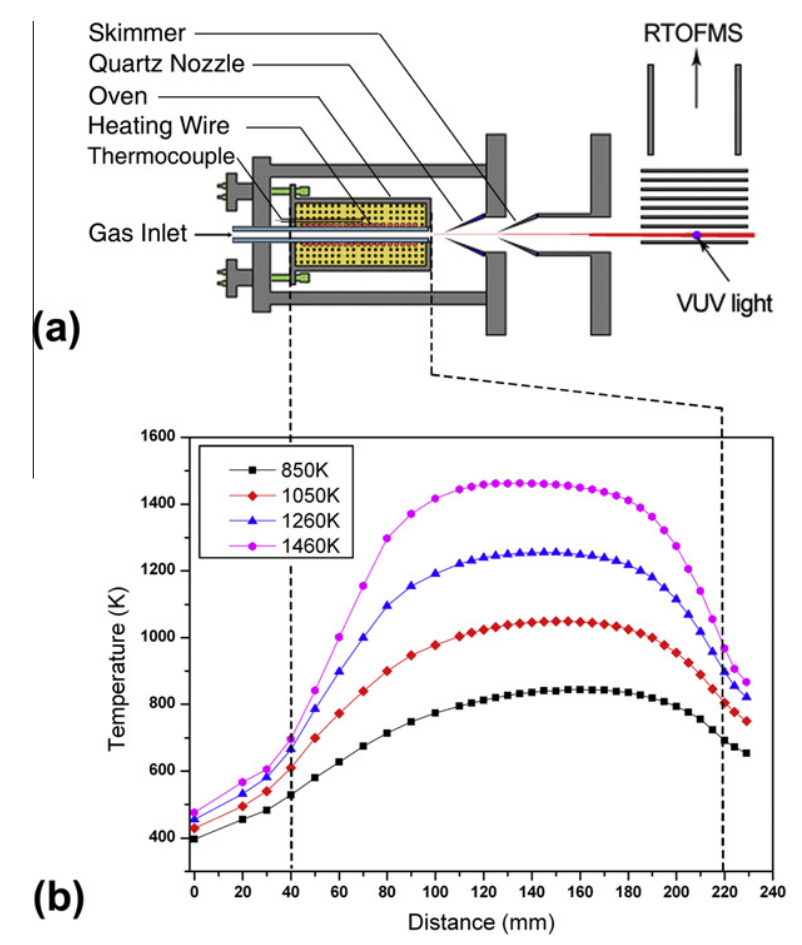

Fig. 1. (a) The schematic diagram of a newly constructed pyrolysis apparatus with molecular-beam sampling mass spectrometer. The red circles inside the furnace denote heating wire with the length of $150 \mathrm{~mm}$ in this work. A tungsten-rhenium (W-Re) thermocouple that connected to a temperature controller was put close to the middle region of the heating wire [40]. (b) The temperature profiles along the centerline of the flow tube were measured by moving a K-type thermocouple (not shown in the figure) from the tube inlet to the sampling point of the quartz nozzle. Four temperature profiles with maximum values of 850, 1050, 1260 and $1460 \mathrm{~K}$ are shown. (For interpretation of the references to colour in this figure legend, the reader is referred to the web version of this article.)

profiles at $T_{\text {out }}=773,973,1173$ and $1373 \mathrm{~K}$ were measured from $d=0-229 \mathrm{~mm}$, as shown in Fig. 1b. Each profile is named by its maximum value $\left(T_{\max }\right)$. The relationship between $T_{\text {out }}$ and $T_{\text {in }}(d)$ at each $d$ can be deduced from the four $T_{\text {in }}(d)$ profiles through second order polynomial fitting. Then, the temperature profiles in the flow tube at other $T_{\text {out }}$ S are plotted, which are provided in Supplemental material. In this work, the maximum value of the profile is used as experimental temperature. The uncertainty of the maximum temperature is estimated to be within $\pm 30 \mathrm{~K}$.

During the experiment, the carrier gas Ar was controlled by a MKS mass flow controller with the flow rate of 0.98 SLM. The liquid flow rate of cyclohexane was set as $0.097 \mathrm{ml} / \mathrm{min}$ (equal to 0.02 SLM in gas phase) using a high performance liquid chromatography pump (Fuli Analytical Instrument Co., Ltd., Zhejiang, China). The initial mole fraction of cyclohexane is 0.02 and the pressure of the pyrolysis chamber was kept as 40 mbar. Cyclohexane was vaporized in a vaporizer heated at a temperature of about $408 \mathrm{~K}$ and mixed with Ar, then was fed into an alumina flow tube (inner diameter: $6.8 \mathrm{~mm}$ ). The sample of cyclohexane $(99.5+\%)$ used in this experiment was purchased from Sinopharm Chemical Reagent Co., Ltd. (Shanghai, China), Ar (99.99\%) was purchased from Nanjing Special Gases Factory Ltd. in China.

Two experimental models were used in this study. One is to measure the photoionization efficiency (PIE) spectra at $1440 \mathrm{~K}$, where the conversion of the reactant is about $80 \%$ and most pyrolysis species have strong signals. The PIE spectra contain precise information of the ionization energies (IEs), which are crucial for species identification. Another one is to measure a series of mass spectra by varying temperature in order to get the mole fractions of Ar, reactant and pyrolysis species. At each temperature, the experiments were 
performed at eight photon energies $(16.65,11.70,11.00,10.50$, $10.00,9.69,9.50$ and $8.86 \mathrm{eV}$ ) in order to implement near threshold measurements. The method to deduce the mole fractions has been reported in detail in our previous work [39], and is briefly described here. For species $i$, the ion signal $\left(S_{i}\right)$ is given by

$$
S_{i}(T, E)=C \times X_{i}(T) \times \sigma_{i}(E) \times D_{i} \times \Phi_{p}(E) \times \lambda(T)
$$

where $C$ is a proportional constant, $X_{i}(T)$ is the mole fraction of species $i$ at the temperature $T, \sigma_{i}(E)$ is the photoionization cross section of the species $i$ at the photon energy $E, D_{i}$ is the mass discrimination factor for the species $i$, and $\Phi_{p}(E)$ is the relative photon flux at the photon energy $E, \lambda(T)$ is the expand factor at temperature $T$, which is derived from the profile of $\mathrm{Ar}$ measured in the pyrolysis process. The units of $T, E, \sigma_{i}(E)$ and $\Phi_{p}(E)$ are $\mathrm{K}, \mathrm{eV}, \mathrm{Mb}$ and $\mathrm{nA}$, respectively.

The mole fraction of pyrolysis species $i$ at temperature $T$ can be obtained via a standard species $s$ at temperature $T_{0}$ by the relationship:

$$
\begin{aligned}
X_{i}(T)= & {\left[S_{i}(T, E) / S_{s}\left(T_{0}, E\right)\right]\left[\sigma_{s}(E) D_{s} / \sigma_{i}(E) D_{i}\right] } \\
& \times\left[\lambda\left(T_{0}\right) / \lambda(T)\right] X_{s}\left(T_{0}\right)
\end{aligned}
$$

Ar was chosen as the standard species to get the mole fractions of $\mathrm{H}_{2}$ and $\mathrm{CH}_{4}$ at $16.65 \mathrm{eV}$. The $\left(\left[\sigma_{i}(E) / \sigma_{\mathrm{Ar}}(E)\right] \times\left[D_{i} / D_{\mathrm{Ar}}\right]\right)$ for $\mathrm{H}_{2}\left(\right.$ or $\left.\mathrm{CH}_{4}\right)$ was gained by cold gas experiments on a binary mixture of $\mathrm{H}_{2}$ (or $\mathrm{CH}_{4}$ ) and $\mathrm{Ar}$ in equal composition at $16.65 \mathrm{eV}$. The mole fraction of cyclohexane was calculated by $X_{\text {cyclohexane }}(T)=\left[S_{\text {cyclohexane }}(T, E) /\right.$ $\left.S_{\text {cyclohexane }}\left(T_{0}, E\right)\right] \times\left[\lambda\left(T_{0}\right) / \lambda(T)\right] \times X_{\text {cyclohexane }}\left(T_{0}\right)$. In this work, $T_{0}$ is $950 \mathrm{~K}$, at which pyrolysis does not happen. $X_{\text {cyclohexane }}\left(T_{0}\right)$ in this temperature equals to its inlet concentration $(0.02)$. To get the mole fractions of other pyrolysis products, cyclohexane was chosen as the standard species at 11.00, 10.50 and $10.00 \mathrm{eV}, 1,3$-butadiene calculated via cyclohexane was used as standard at 9.69 and $9.50 \mathrm{eV}$, and 1,3-cyclohexadiene as standard at $8.86 \mathrm{eV}$. The mole fraction of acetylene was calculated at $11.70 \mathrm{eV}$ using ethylene as a standard. Finally, the mole fraction profiles of each species were normalized in consideration of the mole expansion effect. The errors of the total $\mathrm{C}$ and $\mathrm{H}$ balances for measured species in the whole temperature range are within $10 \%$.

\section{Kinetic modeling}

Kinetic modeling simulations were performed with the Plug Flow Code using Chemkin-PRO software [41]. Most thermodynamic and transport data were taken from JetSurF version 2.0 (JetSurF 2.0) [42], and other data absent in JetSurF 2.0 were taken from the compilation of Goss et al. [43]. Constant pressure of 40 mbar was used in the simulation since there is no big difference for the pressure profiles in the plug flow tube (the inlet pressure is only about 4 mbar higher than that of the outlet part at $1520 \mathrm{~K}$ ). The calibrated temperature profiles were used as input parameters. As shown in Fig. 1, the pyrolysis species were sampled at $229 \mathrm{~mm}$. Therefore the simulated results at $229 \mathrm{~mm}$ are used to compare with the experimental data.

The kinetic model developed in this work is based on our previously published pyrolysis mechanism of three butene isomers [40] and JetSurF 2.0 [42]. The present model consists of 148 species and 557 reactions. Table 1 lists some selected reactions and their rate constants. Figure 2 shows nomenclature, structure and name of some important species related to cyclohexane pyrolysis. The full mechanism is included in Supplemental material. The submechanism of cyclohexane will be discussed below.

\subsection{Reactions of cyclohexane}

Some decomposition channels were proposed for the initial reactions of cyclohexane $\left(\mathrm{cC}_{6} \mathrm{H}_{12}\right)$, which have been reviewed in detail by Kiefer et al. [5]. In their work, both the shock tube experimental and theoretical results confirmed that cyclohexane predominantly isomerizes into 1-hexene $\left(\mathrm{C}_{6} \mathrm{H}_{12}\right)(\mathrm{R} 1)$.

$\mathrm{cC}_{6} \mathrm{H}_{12}=\mathrm{C}_{6} \mathrm{H}_{12}$

None of the proposed molecular channels such as $\mathrm{cC}_{6} \mathrm{H}_{12}=3 \mathrm{C}_{2} \mathrm{H}_{4}$ and $\mathrm{cC}_{6} \mathrm{H}_{12}=\mathrm{cC}_{6} \mathrm{H}_{10}+\mathrm{H}_{2}$ have a significant contribution to the overall dissociation rate. In Kiefer et al.'s work [5], the rate constants of R1 were measured in experiments and calculated by RRKM theory at various pressures $(33,67,133,200$ and 266 mbar) in the temperature range of $1300-2000 \mathrm{~K}$. They suggested that the uncertainty of rate constants is within $\pm 30 \%$ throughout the range of pressure and temperature. In this work, their calculated rate constant at 33 mbar is used and divided by a factor of 1.5 in the simulation to get a better agreement with the experimental measurement.

Besides the $\mathrm{C}-\mathrm{C}$ bond dissociation channel, the $\mathrm{C}-\mathrm{H}$ bond dissociation channel of cyclohexane will produce cyclohexyl radical $\left(\mathrm{cC}_{6} \mathrm{H}_{11}\right)(\mathrm{R} 2)$.

$\mathrm{cC}_{6} \mathrm{H}_{12}=\mathrm{cC}_{6} \mathrm{H}_{11}+\mathrm{H}$

The pressure-dependent rate constants of this reaction (R2) are taken from JetSurF 2.0.

$\mathrm{H}$ abstraction reactions by $\mathrm{H}$ and $\mathrm{CH}_{3}$ attack (R3) and (R4) on cyclohexane are also included in the mechanism with the rate constants taken from the work of Kiefer et al. [5] and JetSurF 2.0, respectively.

$\mathrm{H}+\mathrm{cC}_{6} \mathrm{H}_{12}=\mathrm{cC}_{6} \mathrm{H}_{11}+\mathrm{H}_{2}$
$\mathrm{CH}_{3}+\mathrm{cC}_{6} \mathrm{H}_{12}=\mathrm{cC}_{6} \mathrm{H}_{11}+\mathrm{CH}_{4}$

\subsection{Reactions of 1-hexene}

Kiefer et al. [5] also studied the thermal decomposition of 1hexene. They proposed two main decomposition channels (R5) and (R6) and calculated the rate constants at 33, 67, 133, 200 and 266 mbar. The rate constants at 33 mbar are used in this work.

$\mathrm{C}_{6} \mathrm{H}_{12}=\mathrm{aC}_{3} \mathrm{H}_{5}+n \mathrm{C}_{3} \mathrm{H}_{7}$

$\mathrm{C}_{6} \mathrm{H}_{12}=\mathrm{C}_{4} \mathrm{H}_{7}+\mathrm{C}_{2} \mathrm{H}_{5}$

Besides, the dehydrogenation reactions (R7, R8) and reactions of radicals like $\mathrm{H}$ and $\mathrm{CH}_{3}$ attack on 1-hexene (R9-R18) are included in this model.

\subsection{Reactions of $\mathrm{C}_{6} \mathrm{H}_{11}$ isomers}

Two main reaction channels for cyclohexyl radical $\left(\mathrm{cC}_{6} \mathrm{H}_{11}\right)$ are considered in this model. One channel is the ring-opening isomerization of the cyclohexyl radical to form hex-5-en-1-yl radical $\left(\mathrm{PXC}_{6} \mathrm{H}_{11}\right)$ (R19).

$\mathrm{CC}_{6} \mathrm{H}_{11}=\mathrm{PXC}_{6} \mathrm{H}_{11}$

The hex-5-en-1-yl radical further decomposes to but-3-en-1-yl radical $\left(\mathrm{C}_{4} \mathrm{H}_{7}\right)+\mathrm{C}_{2} \mathrm{H}_{4}(\mathrm{R} 21)$ and isomerizes to hex-1-en-3-yl radical $\left(\mathrm{SAXC}_{6} \mathrm{H}_{11}\right)(\mathrm{R} 22)$.

PXC $_{6} \mathrm{H}_{11}=\mathrm{C}_{4} \mathrm{H}_{7}+\mathrm{C}_{2} \mathrm{H}_{4}$

$\mathrm{PXC}_{6} \mathrm{H}_{11}=\mathrm{SAXC}_{6} \mathrm{H}_{11}$

Then the hex-1-en-3-yl radical mainly decomposes to 1,3-butadiene $\left(\mathrm{C}_{4} \mathrm{H}_{6}\right)$ and ethyl radical $\left(\mathrm{C}_{2} \mathrm{H}_{5}\right)$ through $\beta$-C-C scission (R24).

$\mathrm{SAXC}_{6} \mathrm{H}_{11}=\mathrm{C}_{4} \mathrm{H}_{6}+\mathrm{C}_{2} \mathrm{H}_{5}$

Besides the isomerization channel, the cyclohexyl radical can form cyclohexene $\left(\mathrm{cC}_{6} \mathrm{H}_{10}\right)$ through $\beta-\mathrm{C}-\mathrm{H}$ scission (R20). 
Table 1

Rate constants for selected reactions in the cyclohexane decomposition submechanism, $\mathrm{k}=\mathrm{AT}^{\mathrm{n}} \mathrm{exp}(-E / \mathrm{RT})$. The units are in $\mathrm{cm}{ }^{3}$, mol, s, cal.

\begin{tabular}{|c|c|c|c|c|c|}
\hline No. & Reactions & $A$ & $n$ & E & References \\
\hline \multicolumn{6}{|c|}{ Reactions of cyclohexane } \\
\hline 1 & $\mathrm{cC}_{6} \mathrm{H}_{12}=\mathrm{C}_{6} \mathrm{H}_{12}$ & $1.33 \times 10^{137}$ & -34.70 & 162,775 & {$[5]^{\mathrm{a}}$} \\
\hline 2 & $\mathrm{cC}_{6} \mathrm{H}_{11}+\mathrm{H}(+\mathrm{M})=\mathrm{cC}_{6} \mathrm{H}_{12}(+\mathrm{M})$ & $4.80 \times 10^{13}$ & 0.0 & 0 & {$[42]$} \\
\hline & Low pressure limit & $1.70 \times 10^{58}$ & -12.08 & 11,264 & \\
\hline & Troe parameters: $0.649,1213.1,1213.1,13369.7$ & & & & \\
\hline 3 & $\mathrm{CC}_{6} \mathrm{H}_{12}+\mathrm{H}=\mathrm{cC}_{6} \mathrm{H}_{11}+\mathrm{H}_{2}$ & $2.69 \times 10^{10}$ & 1.4 & 8229 & [5] \\
\hline 4 & $\mathrm{CC}_{6} \mathrm{H}_{12}+\mathrm{CH}_{3}=\mathrm{cC}_{6} \mathrm{H}_{11}+\mathrm{CH}_{4}$ & $3.82 \times 10^{2}$ & 3.2 & 11,634 & [42] \\
\hline \multicolumn{6}{|c|}{ Reactions of 1-hexene } \\
\hline 5 & $\mathrm{C}_{6} \mathrm{H}_{12}=\mathrm{aC}_{3} \mathrm{H}_{5}+n \mathrm{C}_{3} \mathrm{H}_{7}$ & $1.07 \times 10^{80}$ & -19.33 & 95,177 & {$[5]$} \\
\hline 6 & $\mathrm{C}_{6} \mathrm{H}_{12}=\mathrm{C}_{4} \mathrm{H}_{7}+\mathrm{C}_{2} \mathrm{H}_{5}$ & $2.70 \times 10^{80}$ & -19.3 & 107,000 & {$[5]$} \\
\hline 7 & $\mathrm{PXC}_{6} \mathrm{H}_{11}+\mathrm{H}(+\mathrm{M})=\mathrm{C}_{6} \mathrm{H}_{12}(+\mathrm{M})$ & $3.60 \times 10^{13}$ & 0.0 & 0 & {$[42]$} \\
\hline & Low pressure limit & $3.01 \times 10^{48}$ & -9.32 & 5834 & \\
\hline & Troe parameters: $0.498,1314.0,1314.0,50000.0$ & & & & \\
\hline 8 & $\mathrm{SAXC}_{6} \mathrm{H}_{11}+\mathrm{H}(+\mathrm{M})=\mathrm{C}_{6} \mathrm{H}_{12}(+\mathrm{M})$ & $2.00 \times 10^{14}$ & 0.0 & 0 & {$[42]$} \\
\hline & Low pressure limit & $1.33 \times 10^{60}$ & -12.0 & 5968 & \\
\hline & Troe parameters: $0.020,1096.6,1096.6,6859.5$ & & & & \\
\hline 9 & $\mathrm{C}_{6} \mathrm{H}_{12}+\mathrm{H}=\mathrm{C}_{3} \mathrm{H}_{6}+n \mathrm{C}_{3} \mathrm{H}_{7}$ & $3.20 \times 10^{22}$ & -2.39 & 11,180 & {$[42]$} \\
\hline 10 & $\mathrm{C}_{6} \mathrm{H}_{12}+\mathrm{H}=\mathrm{C}_{2} \mathrm{H}_{4}+\mathrm{pC}_{4} \mathrm{H}_{9}$ & $8.00 \times 10^{21}$ & -2.39 & 11,180 & [42] \\
\hline 11 & $\mathrm{C}_{6} \mathrm{H}_{12}+\mathrm{H}=\mathrm{PXC}_{6} \mathrm{H}_{11}+\mathrm{H}_{2}$ & $3.23 \times 10^{-2}$ & 4.70 & 3679 & [42] \\
\hline 12 & $\mathrm{C}_{6} \mathrm{H}_{12}+\mathrm{H}=\mathrm{SXC}_{6} \mathrm{H}_{11}+\mathrm{H}_{2}$ & $3.17 \times 10^{-2}$ & 4.65 & 1340 & {$[42]$} \\
\hline 13 & $\mathrm{C}_{6} \mathrm{H}_{12}+\mathrm{H}=\mathrm{S}_{2} \mathrm{XC}_{6} \mathrm{H}_{11}+\mathrm{H}_{2}$ & $3.17 \times 10^{-2}$ & 4.65 & 1340 & {$[42]$} \\
\hline 14 & $\mathrm{C}_{6} \mathrm{H}_{12}+\mathrm{H}=\mathrm{SAXC}_{6} \mathrm{H}_{11}+\mathrm{H}_{2}$ & $5.40 \times 10^{4}$ & 2.50 & -1900 & [42] \\
\hline 15 & $\mathrm{C}_{6} \mathrm{H}_{12}+\mathrm{CH}_{3}=\mathrm{PXC}_{6} \mathrm{H}_{11}+\mathrm{CH}_{4}$ & $4.50 \times 10^{-1}$ & 3.65 & 7153 & [5] \\
\hline 16 & $\mathrm{C}_{6} \mathrm{H}_{12}+\mathrm{CH}_{3}=\mathrm{SAXC}_{6} \mathrm{H}_{11}+\mathrm{CH}_{4}$ & $2.82 \times 10^{0}$ & 3.60 & 7153 & {$[5]$} \\
\hline 17 & $\mathrm{C}_{6} \mathrm{H}_{12}+\mathrm{CH}_{3}=\mathrm{S} 2 \mathrm{XC}_{6} \mathrm{H}_{11}+\mathrm{CH}_{4}$ & $1.50 \times 10^{0}$ & 3.46 & 5480 & [5] \\
\hline 18 & $\mathrm{C}_{6} \mathrm{H}_{12}+\mathrm{CH}_{3}=\mathrm{SXC}_{6} \mathrm{H}_{11}+\mathrm{CH}_{4}$ & $1.50 \times 10^{0}$ & 3.46 & 5480 & {$[5]$} \\
\hline \multicolumn{6}{|c|}{ Reactions of cyclohexyl radical } \\
\hline 19 & $\mathrm{CC}_{6} \mathrm{H}_{11}(+\mathrm{M})=\mathrm{PXC}_{6} \mathrm{H}_{11}(+\mathrm{M})$ & $6.03 \times 10^{12}$ & 0.07 & 27,983 & {$[42]$} \\
\hline & Low pressure limit & $5.10 \times 10^{-33}$ & 15.29 & -603 & \\
\hline & Troe parameters: $-25.11,225,28,50000.0$ & & & & \\
\hline 20 & $\mathrm{cC}_{6} \mathrm{H}_{11}(+\mathrm{M})=\mathrm{cC}_{6} \mathrm{H}_{10}+\mathrm{H}(+\mathrm{M})$ & $3.34 \times 10^{11}$ & 0.69 & 33,948 & [42] \\
\hline & Low pressure limit & $3.00 \times 10^{-40}$ & 17.33 & -603 & \\
\hline & Troe parameters: $-19.22,230,28,50000.0$ & & & & \\
\hline \multicolumn{6}{|c|}{ Reactions of hexenyl radicals } \\
\hline \multirow[t]{3}{*}{21} & $\mathrm{PXC}_{6} \mathrm{H}_{11}(+\mathrm{M})=\mathrm{C}_{4} \mathrm{H}_{7}+\mathrm{C}_{2} \mathrm{H}_{4}(+\mathrm{M})$ & $3.98 \times 10^{12}$ & 0.12 & 27,572 & [42] \\
\hline & Low pressure limit & $3.30 \times 10^{-43}$ & 18.35 & -603 & \\
\hline & Troe parameters: $-13.87,227,28,50000.0$ & & & & \\
\hline \multirow[t]{3}{*}{22} & $\mathrm{PXC}_{6} \mathrm{H}_{11}(+\mathrm{M})=\mathrm{SAXC}_{6} \mathrm{H}_{11}(+\mathrm{M})$ & $1.55 \times 10^{2}$ & 2.83 & 15,566 & {$[42]$} \\
\hline & Low pressure limit & $1.50 \times 10^{-30}$ & 14.56 & -602 & \\
\hline & Troe parameters: $-13.59,214,28,50000.0$ & & & & \\
\hline \multirow[t]{3}{*}{23} & $\mathrm{PXC}_{6} \mathrm{H}_{11}(+\mathrm{M})=\mathrm{PXCH}_{2} \mathrm{CC}_{5} \mathrm{H}_{9}(+\mathrm{M})$ & $9.55 \times 10^{8}$ & 0.36 & 10,704 & {$[42]$} \\
\hline & Low pressure limit & $2.30 \times 10^{-28}$ & 14.28 & -603 & \\
\hline & Troe parameters: $-18.98,214,28,50000.0$ & & & & \\
\hline \multirow[t]{3}{*}{24} & $\mathrm{SAXC}_{6} \mathrm{H}_{11}(+\mathrm{M})=\mathrm{C}_{4} \mathrm{H}_{6}+\mathrm{C}_{2} \mathrm{H}_{5}(+\mathrm{M})$ & $3.39 \times 10^{11}$ & 0.66 & 32,263 & {$[42]$} \\
\hline & Low pressure limit & $4.00 \times 10^{-42}$ & 18.05 & -603 & \\
\hline & Troe parameters: $-18.50,246,28,50000.0$ & & & & \\
\hline \multirow[t]{3}{*}{25} & $\mathrm{SXC}_{6} \mathrm{H}_{11}(+\mathrm{M})=\mathrm{C}_{3} \mathrm{H}_{6}+\mathrm{aC}_{3} \mathrm{H}_{5}(+\mathrm{M})$ & $4.57 \times 10^{12}$ & 0.13 & 24,386 & {$[42]$} \\
\hline & Low pressure limit & $2.50 \times 10^{-31}$ & 14.57 & -579 & \\
\hline & Troe parameters: $-13.17,268,28,50000.0$ & & & & \\
\hline \multirow[t]{3}{*}{26} & $\mathrm{~S}^{2} \mathrm{XC}_{6} \mathrm{H}_{11}(+\mathrm{M})=\mathrm{C}_{5} \mathrm{H}_{8}-14+\mathrm{CH}_{3}(+\mathrm{M})$ & $8.13 \times 10^{10}$ & 0.78 & 29,648 & {$[42]$} \\
\hline & Low pressure limit & $4.00 \times 10^{-39}$ & 16.78 & -600 & \\
\hline & Troe parameters: $-7.03,314.0,28.0,50000.0$ & & & & \\
\hline 27 & $\mathrm{~S} \mathrm{XC}_{6} \mathrm{H}_{11}(+\mathrm{M})=\mathrm{PXCH}_{2}-3-1 \mathrm{C}_{5} \mathrm{H}_{9}(+\mathrm{M})$ & $7.59 \times 10^{6}$ & 1.81 & 6448 & {$[42]$} \\
\hline & Low pressure limit & $9.30 \times 10^{-19}$ & 11.70 & -603 & \\
\hline & Troe parameters: $-58.54,201,28,50000.0$ & & & & \\
\hline Reac & hexene & & & & \\
\hline 28 & $\mathrm{cC}_{6} \mathrm{H}_{10}=\mathrm{C}_{4} \mathrm{H}_{6}+\mathrm{C}_{2} \mathrm{H}_{4}$ & $2.30 \times 10^{101}$ & -25.30 & 115,500 & {$[44]^{\mathrm{b}}$} \\
\hline 29 & $\mathrm{SAXcC}_{6} \mathrm{H}_{9}+\mathrm{H}(+\mathrm{M})=\mathrm{cC}_{6} \mathrm{H}_{10}(+\mathrm{M})$ & $2.00 \times 10^{14}$ & 0.0 & 0 & \\
\hline & Low pressure limit & $2.66 \times 10^{60}$ & -12.0 & 5968 & \\
\hline 30 & $\mathrm{CC}_{6} \mathrm{H}_{10}+\mathrm{H}=\mathrm{SAXcC}_{6} \mathrm{H}_{9}+\mathrm{H}_{2}$ & $1.10 \times 10^{5}$ & 2.50 & -1900 & {$[61]$} \\
\hline 31 & $\mathrm{CC}_{6} \mathrm{H}_{10}+\mathrm{CH}_{3}=\mathrm{SAXCC}_{6} \mathrm{H}_{9}+\mathrm{CH}_{4}$ & $2.00 \times 10^{11}$ & 0.0 & 7300 & [61] \\
\hline 32 & $\mathrm{SAXcC}_{6} \mathrm{H}_{9}=\mathrm{cC}_{6} \mathrm{H}_{8}-13+\mathrm{H}$ & $1.62 \times 10^{57}$ & -13.0 & 66,036 & [25] \\
\hline 33 & $\mathrm{SAXcC}_{6} \mathrm{H}_{9}=\mathrm{PXC}_{6} \mathrm{H}_{9}-13$ & $5.36 \times 10^{50}$ & -11.9 & 48,276 & {$[25]$} \\
\hline 34 & $\mathrm{SAXcC}_{6} \mathrm{H}_{9}+\mathrm{H}=\mathrm{cC}_{6} \mathrm{H}_{8}-13+\mathrm{H}_{2}$ & $1.80 \times 10^{12}$ & 0.0 & 0 & [42] \\
\hline 35 & $\mathrm{SAXcC}_{6} \mathrm{H}_{9}+\mathrm{CH}_{3}=\mathrm{cC}_{6} \mathrm{H}_{8}-13+\mathrm{CH}_{4}$ & $1.10 \times 10^{13}$ & 0.0 & 0 & [42] \\
\hline 36 & $\mathrm{cC}_{6} \mathrm{H}_{8}-13=\mathrm{SAXCC}_{6} \mathrm{H}_{7}+\mathrm{H}$ & $7.29 \times 10^{40}$ & -8.0 & 87,064 & {$[25]$} \\
\hline 37 & $\mathrm{cC}_{6} \mathrm{H}_{8}-13+\mathrm{H}=\mathrm{SAXCC}_{6} \mathrm{H}_{7}+\mathrm{H}_{2}$ & $1.10 \times 10^{5}$ & 2.5 & -1900 & [61] \\
\hline 38 & $\mathrm{cC}_{6} \mathrm{H}_{8}-13+\mathrm{CH}_{3}=\mathrm{SAXCC}_{6} \mathrm{H}_{7}+\mathrm{CH}_{4}$ & $2.00 \times 10^{12}$ & 0.0 & 7300 & [61] \\
\hline 39 & $\mathrm{SAXcC}_{6} \mathrm{H}_{7}=\mathrm{A} 1+\mathrm{H}$ & $2.64 \times 10^{59}$ & -14.35 & 44,929 & [25] \\
\hline 40 & $\mathrm{SAXcC}_{6} \mathrm{H}_{7}+\mathrm{H}=\mathrm{A} 1+\mathrm{H}_{2}$ & $1.80 \times 10^{12}$ & 0.0 & 0 & [42] \\
\hline 41 & $\mathrm{SAXCC}_{6} \mathrm{H}_{7}+\mathrm{CH}_{3}=\mathrm{A} 1+\mathrm{CH}_{4}$ & $1.10 \times 10^{13}$ & 0.0 & 0 & {$[42]$} \\
\hline
\end{tabular}


Table 1 (continued)

\begin{tabular}{|c|c|c|c|c|c|}
\hline No. & Reactions & $A$ & $n$ & $E$ & References \\
\hline \multicolumn{6}{|c|}{ Reactions of benzene and fulvene } \\
\hline 42 & $\mathrm{aC}_{3} \mathrm{H}_{4}+\mathrm{C}_{3} \mathrm{H}_{3}=\mathrm{A} 1+\mathrm{H}$ & $2.20 \times 10^{11}$ & 0.0 & 2000 & \multirow{2}{*}{$\begin{array}{l}{[62]} \\
\mathrm{d}\end{array}$} \\
\hline 43 & $\mathrm{pC}_{3} \mathrm{H}_{4}+\mathrm{C}_{3} \mathrm{H}_{3}=\mathrm{A} 1+\mathrm{H}$ & $2.20 \times 10^{11}$ & 0.0 & 2000 & \\
\hline 44 & $\mathrm{C}_{3} \mathrm{H}_{3}+\mathrm{C}_{3} \mathrm{H}_{3}=$ fulvene & $7.25 \times 10^{65}$ & -16.0 & 25,035 & [63] \\
\hline 45 & $\mathrm{C}_{3} \mathrm{H}_{3}+\mathrm{C}_{3} \mathrm{H}_{3}=$ fulvene & $4.19 \times 10^{39}$ & -9.0 & 6098 & [63] \\
\hline 46 & $\mathrm{C}_{3} \mathrm{H}_{3}+\mathrm{C}_{3} \mathrm{H}_{3}=\mathrm{A} 1$ & $1.64 \times 10^{66}$ & -15.9 & 27,529 & [63] \\
\hline 47 & $\mathrm{C}_{3} \mathrm{H}_{3}+\mathrm{C}_{3} \mathrm{H}_{3}=\mathrm{A} 1$ & $1.20 \times 10^{35}$ & -7.4 & 5058 & [63] \\
\hline 48 & $\mathrm{C}_{3} \mathrm{H}_{3}+\mathrm{aC}_{3} \mathrm{H}_{5}=$ fulvene $+2 \mathrm{H}$ & $3.26 \times 10^{29}$ & -5.4 & 3390 & [63] \\
\hline 49 & Fulvene $=\mathrm{A} 1$ & $5.62 \times 10^{81}$ & -19.4 & 121,500 & [63] \\
\hline 50 & Fulvene $+\mathrm{H}=\mathrm{A} 1+\mathrm{H}$ & $3.00 \times 10^{12}$ & 0.5 & 2000 & [63] \\
\hline 51 & $n \mathrm{C}_{4} \mathrm{H}_{3}+\mathrm{C}_{2} \mathrm{H}_{3}=\mathrm{A} 1$ & $2.87 \times 10^{14}$ & 0.0 & 817 & [64] \\
\hline 52 & $\mathrm{C}_{4} \mathrm{H}_{4}+\mathrm{C}_{2} \mathrm{H}_{3}=\mathrm{A} 1+\mathrm{H}$ & $1.90 \times 10^{12}$ & 0.0 & 2510 & [65] \\
\hline 53 & $\mathrm{C}_{4} \mathrm{H}_{4}+\mathrm{C}_{2} \mathrm{H}_{2}=\mathrm{A} 1$ & $4.47 \times 10^{11}$ & 0.0 & 30,010 & [55] \\
\hline 54 & $n \mathrm{C}_{4} \mathrm{H}_{5}+\mathrm{C}_{2} \mathrm{H}_{2}=\mathrm{A} 1+\mathrm{H}$ & $2.94 \times 10^{16}$ & -1.09 & 9257 & [66] \\
\hline 55 & $n \mathrm{C}_{4} \mathrm{H}_{5}+\mathrm{C}_{2} \mathrm{H}_{2}=$ fulvene $+\mathrm{H}$ & $1.52 \times 10^{15}$ & -0.76 & 8762 & {$[66]$} \\
\hline 56 & $\mathrm{iC}_{4} \mathrm{H}_{5}+\mathrm{C}_{2} \mathrm{H}_{2}=\mathrm{A} 1+\mathrm{H}$ & $1.47 \times 10^{23}$ & -3.28 & 24,907 & [66] \\
\hline 57 & $\mathrm{iC}_{4} \mathrm{H}_{5}+\mathrm{C}_{2} \mathrm{H}_{2}=$ fulvene $+\mathrm{H}$ & $1.01 \times 10^{34}$ & -5.94 & 28,786 & [66] \\
\hline
\end{tabular}

a Reduced by a factor of 1.5 .

b Reduced by a factor of 8 .

c Refer to $\mathrm{SAXC}_{4} \mathrm{H}_{7}+\mathrm{H}(+\mathrm{M})=1-\mathrm{C}_{4} \mathrm{H}_{8}(+\mathrm{M})$.

d Refer to $\mathrm{aC}_{3} \mathrm{H}_{4}+\mathrm{C}_{3} \mathrm{H}_{3}=\mathrm{A} 1+\mathrm{H}$.
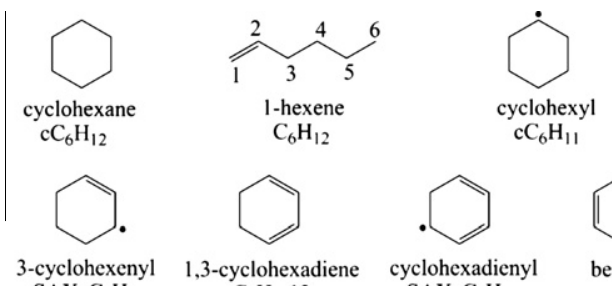

$\mathrm{SAXcC}_{6} \mathrm{H}_{9}$
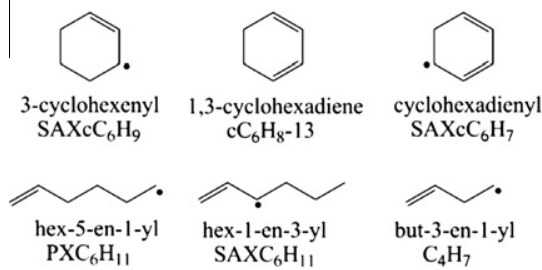
$\mathrm{SAXcC}_{6} \mathrm{H}_{7}$
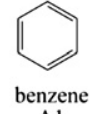

Al
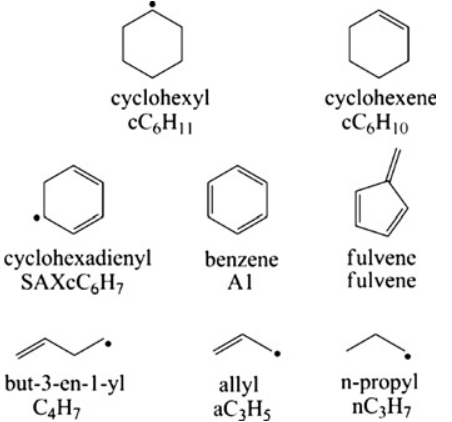
$\mathrm{cC}_{6} \mathrm{H}_{10}$

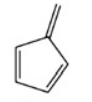

fulvene

fulvene

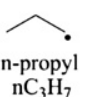

Fig. 2. Nomenclature, structure and name of some important species related to cyclohexane pyrolysis.

$\mathrm{cC}_{6} \mathrm{H}_{11}=\mathrm{cC}_{6} \mathrm{H}_{10}+\mathrm{H}$

The pressure-dependent rate constants of the reactions (R19)(R22), and (R24) are adopted from the JetSurF 2.0.

\subsection{Reactions of cyclohexene}

The dissociation of cyclohexene was studied by Kiefer and Shah [44] in the shock tube over $1200-2000 \mathrm{~K}$ and $147-733$ mbar. The dominant dissociation channel is inverse Diels-Alder molecular elimination to form 1,3-butadiene and ethylene (R28).

$\mathrm{CC}_{6} \mathrm{H}_{10}=\mathrm{C}_{4} \mathrm{H}_{6}+\mathrm{C}_{2} \mathrm{H}_{4}$

Rate constant reported by Kiefer and Shah [44] in 147-733 mbar is chosen and reduced by a factor of 8 in this model.

On the other hand, the stepwise dehydrogenation of cyclohexene can form benzene.

$$
\begin{aligned}
& \mathrm{SAXcC}_{6} \mathrm{H}_{9}+\mathrm{H}=\mathrm{cC}_{6} \mathrm{H}_{10} \\
& \mathrm{cC}_{6} \mathrm{H}_{10}+\mathrm{H}=\mathrm{SAXcC}_{6} \mathrm{H}_{9}+\mathrm{H}_{2} \\
& \mathrm{SAXcC}_{6} \mathrm{H}_{9}=\mathrm{cC}_{6} \mathrm{H}_{8}-13+\mathrm{H} \\
& \mathrm{cC}_{6} \mathrm{H}_{8}-13=\mathrm{SAXcC}_{6} \mathrm{H}_{7}+\mathrm{H} \\
& \mathrm{CC}_{6} \mathrm{H}_{8}-13+\mathrm{H}=\mathrm{SAXCC}_{6} \mathrm{H}_{7}+\mathrm{H}_{2} \\
& \mathrm{SAXcC}_{6} \mathrm{H}_{7}=\mathrm{A} 1+\mathrm{H}
\end{aligned}
$$

Rate constants of the above reactions are listed in Table 1.

\subsection{Reactions of cyclopentadiene and aromatics}

To simulate the formation of aromatics, such as benzene, toluene, phenylacetylene, styrene, ethylbenzene and indene detected in this work, aromatics reactions taken from the pyrolysis model of toluene [45] are also included, which have been validated by the low pressure ( 40 mbar) premixed flame data of toluene [46] and ethylbenzene [47].

$\mathrm{aC}_{3} \mathrm{H}_{4}+\mathrm{C}_{3} \mathrm{H}_{3}=\mathrm{A} 1+\mathrm{H}$

$\mathrm{pC}_{3} \mathrm{H}_{4}+\mathrm{C}_{3} \mathrm{H}_{3}=\mathrm{A} 1+\mathrm{H}$

$\mathrm{C}_{3} \mathrm{H}_{3}+\mathrm{C}_{3} \mathrm{H}_{3}=$ fulvene

$\mathrm{C}_{3} \mathrm{H}_{3}+\mathrm{C}_{3} \mathrm{H}_{3}=\mathrm{A} 1$

$\mathrm{C}_{3} \mathrm{H}_{3}+\mathrm{aC}_{3} \mathrm{H}_{5}=$ fulvene $+2 \mathrm{H}$

The rate constants of $\mathrm{C}_{3} \mathrm{H}_{3}+\mathrm{C}_{3} \mathrm{H}_{3}$ to form phenyl $+\mathrm{H}$, benzene and fulvene used in this work are based on the work of Miller and Klippenstein [48] and Georgievskii et al. [49]. In Georgievskii et al.'s work, the total rate constant of recombination of $\mathrm{C}_{3} \mathrm{H}_{3}$ was compared with available experimental data, such as the work of Scherer et al. [50] and Fernandes et al. [51], showing an excellent agreement between experiments and calculations. Besides these $\mathrm{C} 3+\mathrm{C} 3$ channels, the $\mathrm{C} 4+\mathrm{C} 2$ channels and the isomerization reactions of fulvene and benzene are also considered, as shown in Table 1.

Some groups have studied the pyrolysis of cyclopentadiene $\left(\mathrm{C}_{5} \mathrm{H}_{6}\right)$, such as Roy et al. [52] and Bacskay and Mackie [53]. The submechanism of $\mathrm{C}_{5} \mathrm{H}_{6}$ in this work is taken from the USC Mech II [54], the low-pressure soot model developed by Richter et al. [55] and the $\mathrm{C}_{5} \mathrm{H}_{6}$ mechanism proposed by Bacskay and Mackie [53]. These reactions had been included in the pyrolysis model of toluene [45].

\subsection{Reactions of C4-C1 species}

The C4-C1 mechanism used in this work have been validated by our pyrolysis study of three butene isomers [40]. The reactions related to butene isomers have been discussed in detail in previous work, and will not be described here. The mechanism of 1,3-butadiene is adopted from USC Mech II [54], which have been validated by various kinds of experimental data in the work of Laskin et al. 
Table 2

List of species detected in the pyrolysis of cyclohexane.

\begin{tabular}{|c|c|c|c|c|c|c|c|c|}
\hline$m / z$ & Formula & Species & Photon energy $(\mathrm{eV})$ & PICS (Mb) & $T_{\text {Form }}(\mathrm{K})$ & $T_{\mathrm{M}}(\mathrm{K})$ & $X_{\mathrm{M}}$ & Refs. of PICS \\
\hline 2 & $\mathrm{H}_{2}$ & Hydrogen ${ }^{a}$ & e & e & 1230 & 1520 & $2.37 \mathrm{E}-2$ & e \\
\hline 15 & $\mathrm{CH}_{3}$ & Methyl radical ${ }^{\mathrm{b}}$ & 10.00 & 5.6 & 1150 & 1460 & $3.50 \mathrm{E}-4$ & [67] \\
\hline 16 & $\mathrm{CH}_{4}$ & Methane $^{\mathrm{a}}$ & e & e & 1230 & 1520 & $5.40 \mathrm{E}-3$ & e \\
\hline 26 & $\mathrm{C}_{2} \mathrm{H}_{2}$ & Acetylene ${ }^{\mathrm{b}}$ & 11.70 & 24.9 & 1310 & 1520 & $6.43 E-3$ & [68] \\
\hline 28 & $\mathrm{C}_{2} \mathrm{H}_{4}$ & Ethylene $^{\mathrm{b}}$ & 11.00 & 7.8 & 1200 & 1520 & $2.00 \mathrm{E}-2$ & [68] \\
\hline 39 & $\mathrm{C}_{3} \mathrm{H}_{3}$ & Propargyl radical ${ }^{\mathrm{c}}$ & 10.50 & 9.0 & 1310 & 1520 & $4.81 \mathrm{E}-4$ & [69] \\
\hline \multirow[t]{2}{*}{40} & $\mathrm{C}_{3} \mathrm{H}_{4}$ & Allene ${ }^{\mathrm{b}}$ & 10.00 & 5.7 & 1230 & 1510 & $1.26 \mathrm{E}-3$ & [70] \\
\hline & & Propyne ${ }^{b}$ & 10.50 & 23.1 & 1280 & 1520 & $2.26 \mathrm{E}-3$ & [68] \\
\hline 41 & $\mathrm{C}_{3} \mathrm{H}_{5}$ & Allyl radical $^{\mathrm{c}}$ & 9.50 & 5.6 & 1150 & 1360 & $4.55 \mathrm{E}-4$ & [71] \\
\hline 42 & $\mathrm{C}_{3} \mathrm{H}_{6}$ & Propene ${ }^{\mathrm{b}}$ & 10.50 & 11.1 & 1230 & 1440 & $2.76 \mathrm{E}-3$ & [72] \\
\hline 50 & $\mathrm{C}_{4} \mathrm{H}_{2}$ & 1,3-Butadiyne ${ }^{\mathrm{b}}$ & 10.50 & 23.8 & 1410 & 1520 & $1.52 \mathrm{E}-4$ & [68] \\
\hline 52 & $\mathrm{C}_{4} \mathrm{H}_{4}$ & Vinylacetylene $^{\mathrm{b}}$ & 10.50 & 22.8 & 1310 & 1520 & $1.32 \mathrm{E}-3$ & [68] \\
\hline 54 & $\mathrm{C}_{4} \mathrm{H}_{6}$ & 1,3-Butadiene ${ }^{b}$ & 11.00 & 24.2 & 1200 & 1440 & $6.64 \mathrm{E}-3$ & \\
\hline \multirow[t]{2}{*}{56} & $\mathrm{C}_{4} \mathrm{H}_{8}$ & 1-Butene ${ }^{b}$ & 10.50 & 10.0 & 1200 & 1360 & $8.73 \mathrm{E}-4$ & [73] \\
\hline & & 2-Butene ${ }^{c}$ & 9.50 & 5.2 & 1240 & 1460 & $7.84 \mathrm{E}-5$ & [74] \\
\hline 64 & $\mathrm{C}_{5} \mathrm{H}_{4}$ & 1,3-Pentadiyne ${ }^{\mathrm{d}}$ & 10.00 & 33.2 & 1360 & 1520 & $2.51 \mathrm{E}-5$ & [75] \\
\hline 65 & $\mathrm{C}_{5} \mathrm{H}_{5}$ & Cyclopentadienyl radical $^{\mathrm{d}}$ & 10.00 & 9.1 & 1310 & 1520 & $1.33 \mathrm{E}-4$ & est \\
\hline 66 & $\mathrm{C}_{5} \mathrm{H}_{6}$ & Cyclopentadiene ${ }^{\mathrm{d}}$ & 9.50 & 15.7 & 1260 & 1520 & $4.61 \mathrm{E}-4$ & [75] \\
\hline 68 & $\mathrm{C}_{5} \mathrm{H}_{8}$ & $\begin{array}{l}\text { 1,3-Pentadiene }{ }^{c} \\
\text { Isoprene }\end{array}$ & 9.50 & 12.7 & 1200 & 1390 & $3.37 \mathrm{E}-4$ & [70] \\
\hline 70 & $\mathrm{C}_{5} \mathrm{H}_{10}$ & 1-Pentene ${ }^{b}$ & 10.00 & 14.4 & 1200 & 1390 & $2.19 E-5$ & {$[74]$} \\
\hline 76 & $\mathrm{C}_{6} \mathrm{H}_{4}$ & Benzyne $^{d}$ & 9.50 & 27.0 & 1410 & 1520 & $2.27 \mathrm{E}-5$ & est \\
\hline \multirow[t]{2}{*}{78} & $\mathrm{C}_{6} \mathrm{H}_{6}$ & Benzene ${ }^{b}$ & 9.50 & 11.05 & 1330 & 1520 & $6.34 \mathrm{E}-4$ & [68] \\
\hline & & Fulvene ${ }^{d}$ & 8.86 & 3.9 & 1330 & 1490 & $9.72 \mathrm{E}-5$ & est \\
\hline 80 & $\mathrm{C}_{6} \mathrm{H}_{8}$ & 1,3-Cyclohexadiene ${ }^{b}$ & 9.50 & 18.3 & 1230 & 1450 & $1.27 \mathrm{E}-4$ & [74] \\
\hline \multirow[t]{2}{*}{82} & $\mathrm{C}_{6} \mathrm{H}_{10}$ & Cyclohexene $^{\mathrm{b}}$ & 9.50 & 8.9 & 1180 & 1330 & $2.13 \mathrm{E}-4$ & [74] \\
\hline & & 1,3-Hexadiene ${ }^{c}$ & 8.86 & 8.7 & 1230 & 1390 & $1.82 \mathrm{E}-5$ & [74] \\
\hline \multirow[t]{2}{*}{84} & $\mathrm{C}_{6} \mathrm{H}_{12}$ & Cyclohexane & & & & & & [76] \\
\hline & & 1-Hexene ${ }^{c}$ & 9.69 & 3.7 & 1150 & 1310 & $1.31 \mathrm{E}-4$ & [70] \\
\hline 92 & $\mathrm{C}_{7} \mathrm{H}_{8}$ & Toluene $^{\mathrm{b}}$ & 9.50 & 18.5 & 1360 & 1520 & $7.70 \mathrm{E}-5$ & [77] \\
\hline 102 & $\mathrm{C}_{8} \mathrm{H}_{6}$ & Phenylacetylene ${ }^{\mathrm{b}}$ & 9.50 & 29.4 & 1410 & 1520 & $2.00 \mathrm{E}-5$ & [77] \\
\hline 104 & $\mathrm{C}_{8} \mathrm{H}_{8}$ & Styrene ${ }^{b}$ & 9.50 & 26.3 & 1390 & 1520 & $4.34 \mathrm{E}-5$ & [77] \\
\hline 106 & $\mathrm{C}_{8} \mathrm{H}_{10}$ & Ethylbenzene ${ }^{\mathrm{b}}$ & 10.00 & 25.8 & 1330 & 1520 & $1.65 \mathrm{E}-5$ & [77] \\
\hline 116 & $\mathrm{C}_{9} \mathrm{H}_{8}$ & Indene ${ }^{b}$ & 10.50 & 52.2 & 1410 & 1520 & $1.69 \mathrm{E}-5$ & [77] \\
\hline
\end{tabular}

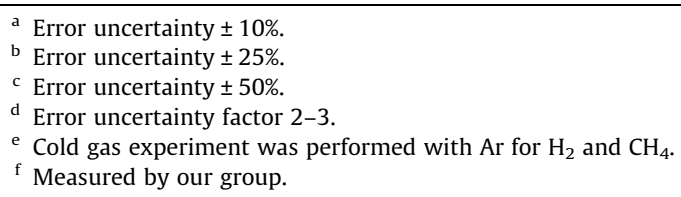

[56]. The submechanism of $\mathrm{C}_{3} \mathrm{H}_{5}$ and $\mathrm{C}_{3} \mathrm{H}_{4}$ is mainly taken from USC Mech II [54], the work of Kiefer et al. [5] and the calculation results of Miller et al. [57]. For the reactions of $\mathrm{H}$ with propyne, the theoretical study by Miller et al. [57] showed a remarkably good agreement with the shock tube experiments of Bentz et al. [58]. The reactions related with these C4-C3 species have been given in Ref. [40] and are not described here. The mechanism of C2-C1 is adopted from USC Mech II [54].

\section{Results and discussion}

In this work, more than 30 species were detected and identified, as listed in Table 2 with initial formation temperature $\left(T_{\text {Form }}\right), T_{\max }$ and concentration at $T_{\max }$ of each product. The literatures of photoionization cross sections (PICSs) and the value used for mole fraction evaluation are also given in Table 2. PICSs of most species are derived from the literatures. PICS of 1,3-butadiene measured by our group is used for calculating its mole fraction in this work. For species with unknown values, their PICSs are estimated from molecules with similar structures and IEs. The uncertainties of the measured mole fractions of each species are also given in Table 2. In this work, the uncertainties for $\mathrm{H}_{2}$ and $\mathrm{CH}_{4}$ are $\pm 10 \%$. For most species with the known PICSs, the uncertainties are $\pm 25-50 \%$. The uncertainties for species with unknown PICSs are estimated to be a factor of 2-3.

The experimental (open symbols) and simulated results (solid lines) of cyclohexane pyrolysis are shown in Fig. 3. In general, the model reproduces the initial formation temperatures, peak temperatures and concentrations of most species, which validates the accuracy of this model. We will discuss the reaction flux of the reactant, the formation of benzene and larger aromatics based on the simulation results below.

\subsection{Consumption of cyclohexane}

Figure 4 displays the ROP analysis of cyclohexane consumption at 1360 and $1520 \mathrm{~K}$, respectively. The reason for choosing these two temperatures is that the consumption of the reactant is about $50 \%$ at $1360 \mathrm{~K}$ and almost $100 \%$ at $1520 \mathrm{~K}$. As can be seen from Fig. 3a, the simulation reproduces well the consumption of cyclohexane. The ROP analysis in Fig. 4a shows that cyclohexane is consumed via two channels at $1360 \mathrm{~K}$, i.e. the unimolecular dissociation (R1, $36 \%$ ) to form 1-hexene, and $\mathrm{H}$ loss by radical attack to form cyclohexyl radical (R3 and R4, 64\%). The sensitivity analysis of cyclohexane is carried out at $1360 \mathrm{~K}$, as shown in Fig. 5. It reveals that R1 and R3 have large negative coefficient for cyclohexane, that is to say, increasing the pre-exponential factors (A) of R1 and R3 will promote the consumption of cyclohexane. The good agreement between simulation and experiment indicates the reasonability of rate constants of $\mathrm{R} 1$ and $\mathrm{R} 3$ used in this work.

As mentioned above, some researchers proposed that the isomerization of cyclohexane to 1-hexene is the initial decomposition channel $[5,29,30]$. In this work, 1 -hexene was observed and identified. As shown in Fig. 6, two onsets are observed: one is located at 


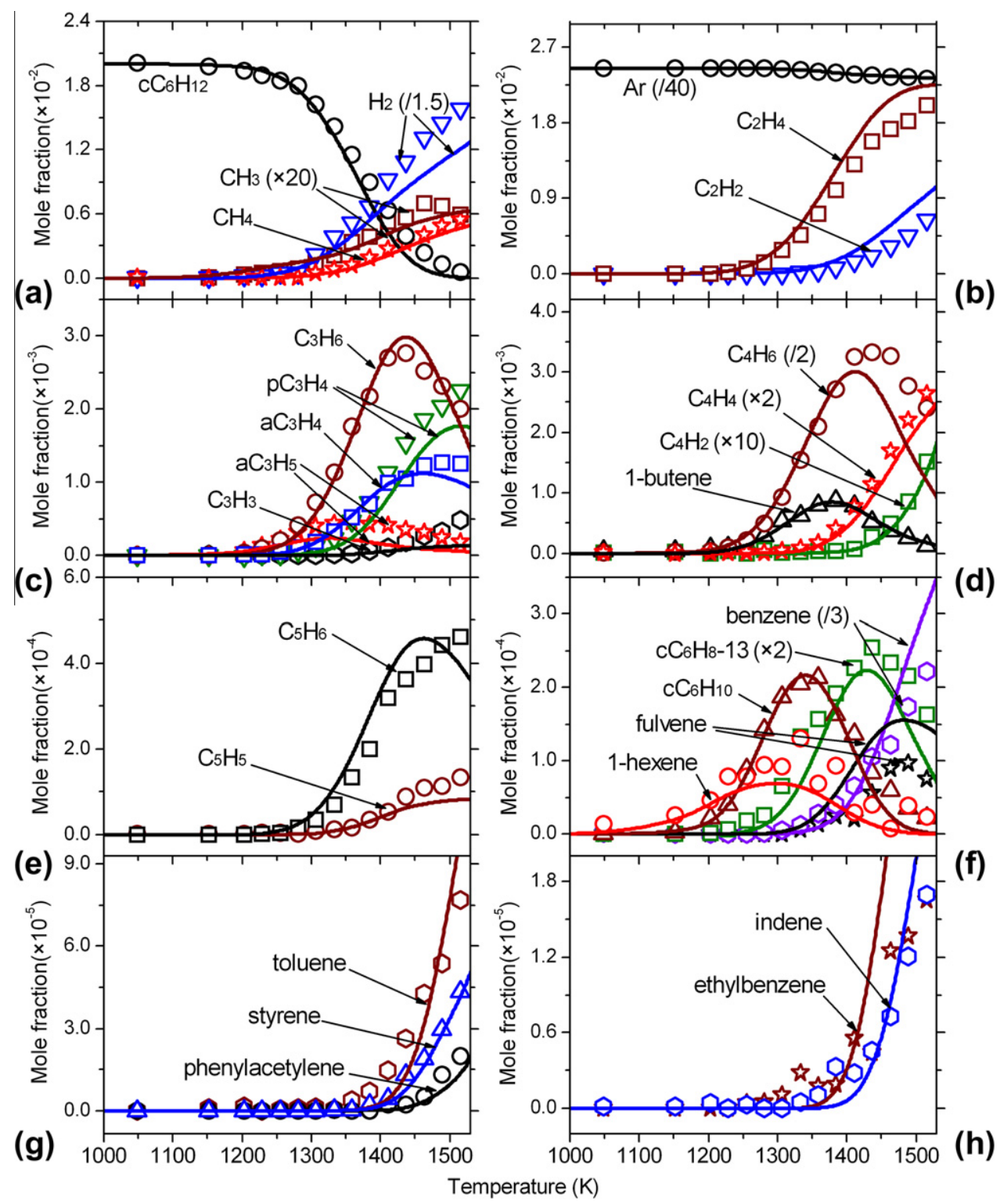

Fig. 3. Experimental (open symbols) and simulated (solid lines) mole fractions of cyclohexane, Ar and pyrolysis products.

$9.86 \mathrm{eV}$, corresponding to cyclohexane; the other is $9.47 \mathrm{eV}$, which corresponds to 1 -hexene. The measurement of 1 -hexene is very important to clarify the initial decomposition channels of cyclohexane pyrolysis at the low-pressure condition. To obtain the mole fraction of 1-hexene, the medium photon energy $(9.69 \mathrm{eV})$ between the IEs of 1-hexene and cyclohexane was chosen, which is about $0.2 \mathrm{eV}$ above the IE of 1-hexene. The slightly weak signal of 1-hexene is mainly resulted from its weak PICS at this energy. It can be seen from Fig. 3f that 1-hexene begins to be produced at about $1150 \mathrm{~K}$ and exhibits a maximum at about $1310 \mathrm{~K}$. The lowest formation temperature of 1-hexene confirms that cyclohexane firstly isomerizes to 1-hexene. Our model reproduces the experimental result of 1 -hexene within the experimental uncertainty. 1-Hexene is mainly decomposed by C3-C4 bond dissociation to form the allyl radical $\left(\mathrm{aC}_{3} \mathrm{H}_{5}\right)$ and $n$-propyl radical $\left(n \mathrm{C}_{3} \mathrm{H}_{7}\right)$ (R5, 93\%). Small amount of 1 -hexene is consumed by $\mathrm{C} 4-\mathrm{C} 5$ bond dissociation to form ethyl and but-3-en-1-yl radical (R6, 3\%). Figure 7 presents the sensitivity analysis of 1 -hexene at $1310 \mathrm{~K}$. It can be seen that R1 has the largest positive coefficient, while R5 has the largest negative coefficient for 1-hexene (the coefficient for R5 is about -1.26). When the pre-exponential factor (A) of R5 is divided by a factor of 2 , the concentration of 1 -hexene is 2.4 times larger than the original one.

The formed $n$-propyl radical rapidly dissociates through two channels: $\beta-\mathrm{C}-\mathrm{C}$ bond dissociation to form methyl radical and ethylene, and $\beta-\mathrm{C}-\mathrm{H}$ scission to form propene. The former channel contributes about $80 \%$ of the total dissociation of the $n$-propyl radical. There exist three channels for the consumption of the allyl radical: addition of the methyl radical to form 1-butene (36\%), $\beta$ $\mathrm{C}-\mathrm{H}$ scission to form allene (21\%) and addition of hydrogen radical to form propene (32\%). The allyl radical is detected and quantified in this work. As can be seen from Fig. 3c, the initial formation temperature of the allyl radical is about $1150 \mathrm{~K}$ and reaches to a maximum at about $1360 \mathrm{~K}$. The relation of the allyl radical with 1hexene is in agreement with the experimental observation that the allyl radical has the similar shape with 1-hexene. ROP analysis (not shown in Fig. 4) shows that 1-butene, allene and propene are mainly formed from the allyl radical through the reactions mentioned above. For propene, the $\beta-\mathrm{C}-\mathrm{H}$ scission of the $n$-propyl radical also has some contribution to its formation. Most allene is consumed through $\mathrm{H}$-assisted isomerization and direct isomerization reaction to form propyne. The initial formation temperature of 


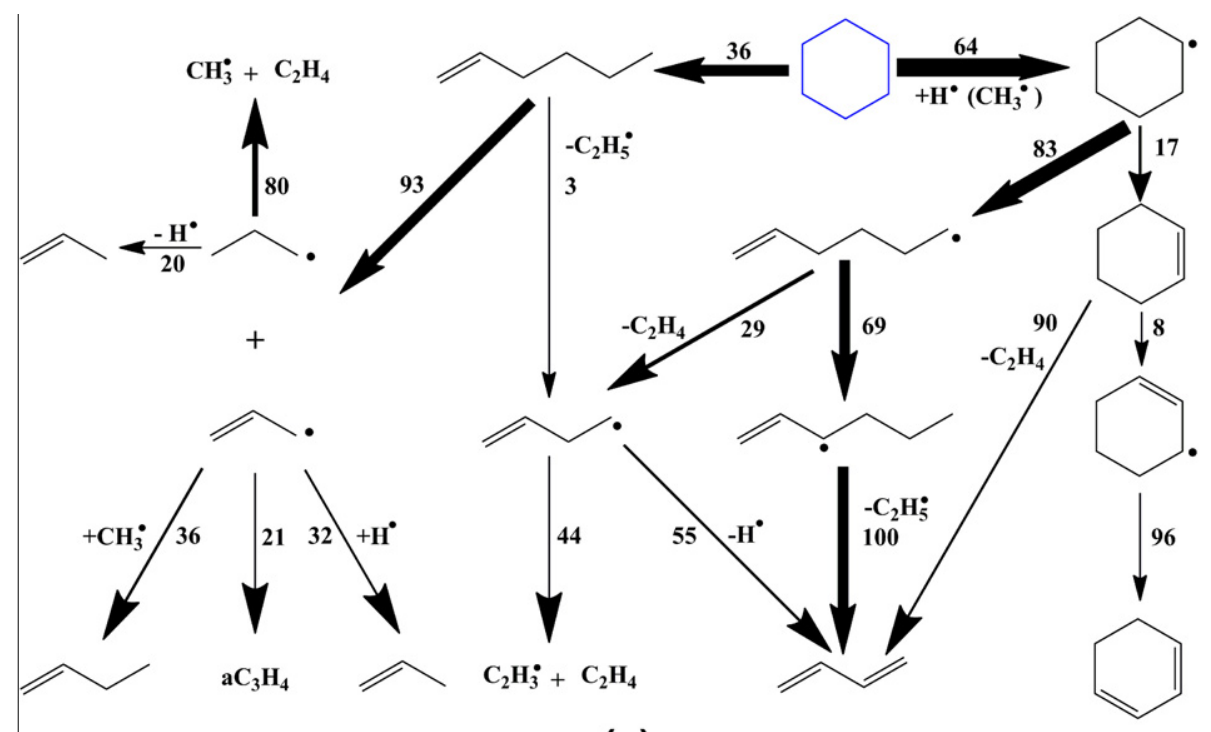

(a)

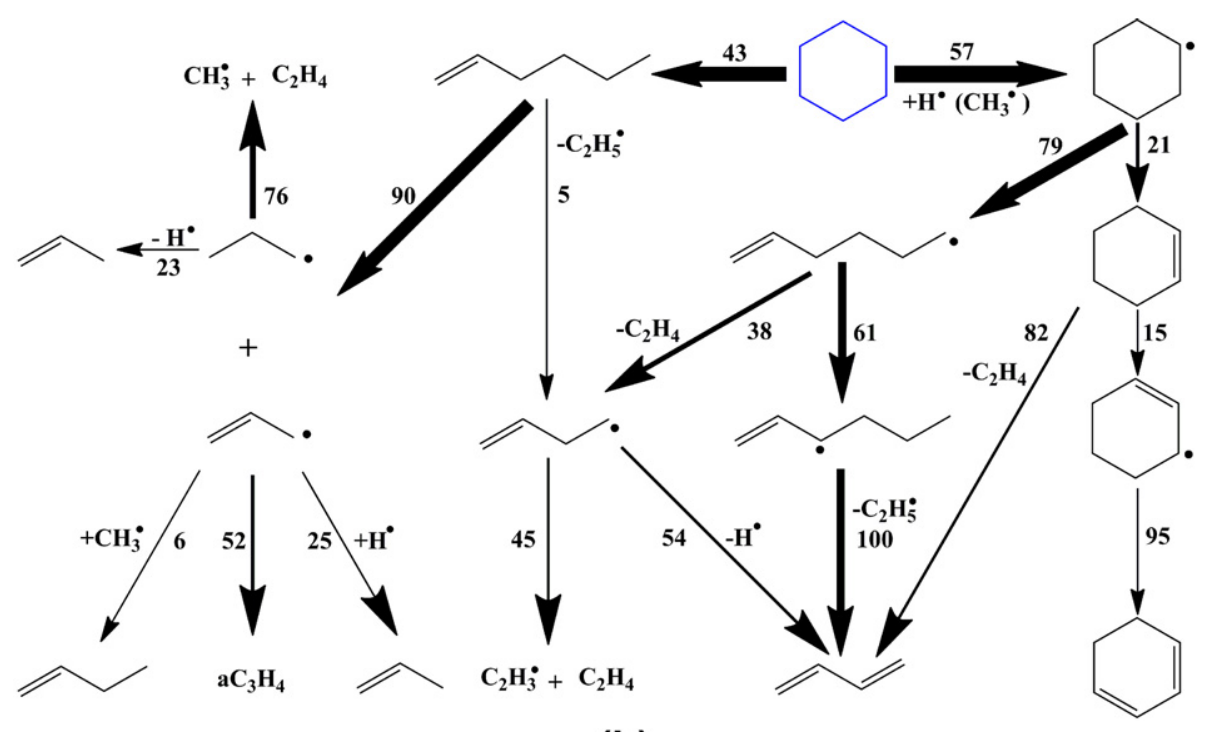

(b)

Fig. 4. ROP analysis of cyclohexane at (a) $1360 \mathrm{~K}$ and (b) $1520 \mathrm{~K}$, respectively. The numbers indicate the conversion percentage.

allene is about $50 \mathrm{~K}$ lower than that of propyne, while the concentration of propyne is twice as much as allene. In a word, the simulated mole fraction profiles of $\mathrm{C} 3$ products and 1-butene in Fig. 3c and $\mathrm{d}$ agree with the experimental results.

On the other hand, the formed cyclohexyl radical is consumed via two channels: isomerization to form hex-5-en-1-yl radical (R19, 83\%) and $\beta-\mathrm{C}-\mathrm{H}$ scission to produce cyclohexene (R20, $17 \%)$. The hex-5-en-1-yl radical is mainly consumed by $1,4-\mathrm{H}$ shift isomerization to form hex-1-en-3-yl radical (R22, 69\%), which further decomposes to 1,3-butadiene and ethyl radical (R24, 100\%). The rest of the hex-5-en-1-yl radical is mostly consumed by $\beta$-CC scission to form ethylene and but-3-en-1-yl radical (R21, 29\%). Further reactions of the but-3-en-1-yl radical include two channels: $\beta-\mathrm{C}-\mathrm{H}$ scission to produce 1,3 -butadiene (55\%) and $\beta-\mathrm{C}-\mathrm{C}$ scission to the vinyl radical and ethylene (44\%). As mentioned above, cyclohexene is consumed mainly by inverse Diels-Alder molecular elimination to form 1,3-butadiene and ethylene (R28, $90 \%$ ). The reactions of $\mathrm{H}$-loss by $\beta-\mathrm{C}-\mathrm{H}$ scission (R29) and radical attack (R30) to produce 3-cyclohexenyl radical only account for about $8 \%$ of the consumption of cyclohexene. The stepwise dehydrogenation of the 3-cyclohexenyl radical forms benzene via 1,3-cyclohexadiene and cyclohexadienyl (not shown in Fig. 4). This is in agreement with the experimental observation that the initial formation temperature is cyclohexene $<1,3$-cyclohexadiene $<$ benzene. The simulated cyclohexene and 1,3-cyclohexadiene mole fraction profiles agree well with the experimental results. It should be noted that the simulated concentrations of cyclohexene and 1,3cyclohexadiene are sensitive to the reaction $\mathrm{cC}_{6} \mathrm{H}_{10}=\mathrm{C}_{4} \mathrm{H}_{6}+\mathrm{C}_{2} \mathrm{H}_{4}$ (R28). As mentioned in Section 3.4, the rate constant of $\mathrm{R} 28$ taken from Kiefer and Shah [44] is reduced by a factor of 8 . If there is no reduction of this rate, the simulated concentrations of cyclohexene and 1,3-cyclohexadiene will be underpredicted by a factor of 6 and 8 , respectively. Considering the reliability of measured cyclohexene and 1,3-cyclohexadiene concentration using known PICSs, we think that the rate constant of R28 used in this work is reasonable under our experimental condition. For the ROP analysis at $1520 \mathrm{~K}$, as shown in Fig. 4b, more 1-hexene (43\%) is produced by cyclohexane comparing with Fig. 4a at $1360 \mathrm{~K}$. Further reactions of 1-hexene and the cyclohexyl radical are similar with reactions at $1360 \mathrm{~K}$. The only discrepancy occurs on the allyl radical. The allyl radical is 


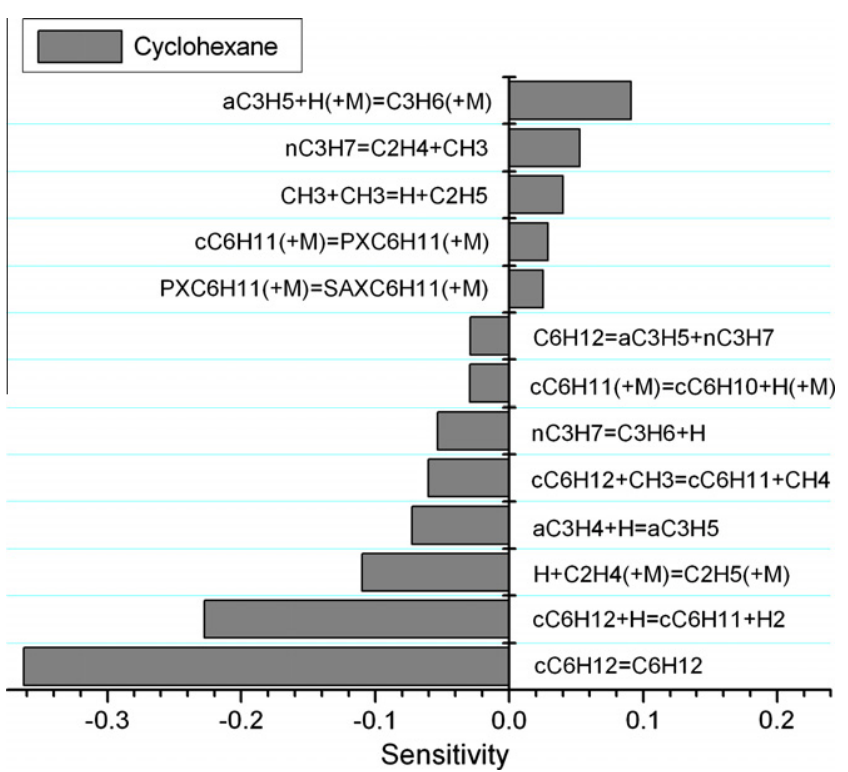

Fig. 5. Sensitivity analysis of cyclohexane at $1360 \mathrm{~K}$. Reactions with absolute sensitivity coefficients larger than 0.025 are considered.

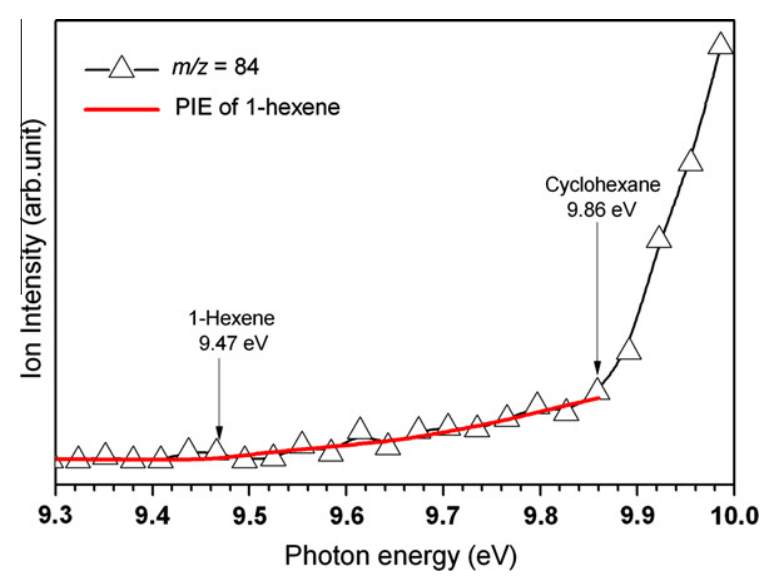

Fig. 6. PIE spectra of $m / z 84$ measured in the pyrolysis of cyclohexane at $1440 \mathrm{~K}$. The red solid line is the PIE of 1-hexene measured by Yang et al. [70].

consumed mainly by $\beta$-C-H scission to form allene (52\%), and contribution via addition reactions to form propene (25\%) and 1-butene $(6 \%)$ is small.

Basing on Fig. 4a and b, we can draw the conclusion that cyclohexane is consumed mainly through the reaction sequence of cyclohexane $\rightarrow 1$-hexene $\rightarrow$ allyl radical $+n$-propyl radical and cyclohexane $\rightarrow$ cyclohexyl radical $\rightarrow$ hex-5-en-1-yl, which further decomposes to 1,3-butadiene via the hex-1-en-3-yl and but-3en-1-yl radicals. It can be seen that most carbon flux flows into 1,3-butadiene, which explains the extremely high mole fraction of 1,3-butadiene $\left(6.64 \times 10^{-3}\right)$ in the experimental measurement. The model reproduces the mole fraction profile of 1,3-butadiene within the experimental uncertainty. Most 1,3-butadiene is consumed by $\mathrm{H}$ attack followed by $\beta-\mathrm{C}-\mathrm{C}$ scission to form ethylene and vinyl radical, while a small amount is consumed by radical attack to form $\mathrm{iC}_{4} \mathrm{H}_{5}$ and $n \mathrm{C}_{4} \mathrm{H}_{5}$, which is the source of vinylacetylene and 1,3-butadiyne, as shown in Fig. 3d.

\subsection{Formation of cyclopentadiene, benzene and other aromatics}

In this work, two five-member-ring products were identified and quantified, i.e. cyclopentadiene and cyclopentadienyl radical,

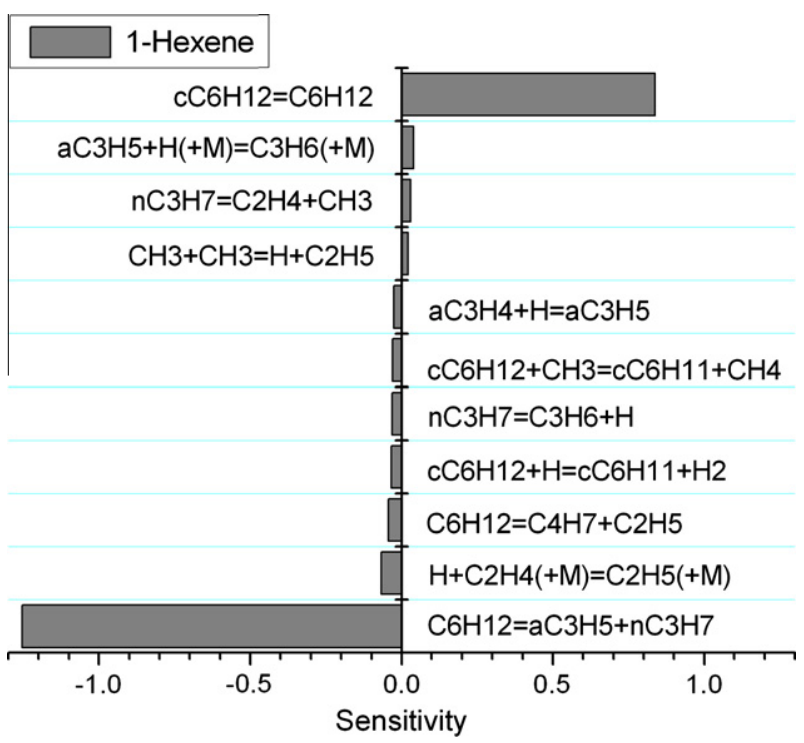

Fig. 7. Sensitivity analysis of 1 -hexene at $1310 \mathrm{~K}$. Reactions with absolute sensitivity coefficients larger than 0.02 are considered.

as shown in Fig. 3e. The ROP analysis at $1520 \mathrm{~K}$ shows that cyclopentadiene is mainly formed from the reaction sequence $\mathrm{aC}_{3} \mathrm{H}_{5}+\mathrm{C}_{2} \mathrm{H}_{3} \rightarrow \mathrm{lC}_{5} \mathrm{H}_{7} \rightarrow \mathrm{C}_{5} \mathrm{H}_{6}$ and $\mathrm{iC}_{4} \mathrm{H}_{5}+\mathrm{CH}_{3} \rightarrow \mathrm{IC}_{5} \mathrm{H}_{7} \rightarrow \mathrm{C}_{5} \mathrm{H}_{6}$. $\mathrm{H}$-abstraction of cyclopentadiene is the major source of the cyclopentadienyl radical, which dominantly dissociates to $\mathrm{C}_{2} \mathrm{H}_{2}$ and $\mathrm{C}_{3} \mathrm{H}_{3}$.

Formation of the first aromatics ring such as benzene and phenyl radical has been thought to be the rate controlling step of PAH and soot formation. A recent study by Hansen et al. [59] revealed that benzene formation is affected by the fuel structure in premixed flames of isomeric $\mathrm{C}_{6} \mathrm{H}_{12}$ fuels. They suggested that benzene is formed dominantly by $\mathrm{C}_{3}+\mathrm{C}_{3}$ reactions, i.e. $\mathrm{C}_{3} \mathrm{H}_{3}+\mathrm{C}_{3} \mathrm{H}_{3}$ and $\mathrm{C}_{3} \mathrm{H}_{3}+\mathrm{aC}_{3} \mathrm{H}_{5}$ in the 1-hexene flame since 1-hexene decomposes dominantly to $\mathrm{C} 3$ products, which had been confirmed later by the simulation results [60]. However, in the cyclohexane flame, they suggested that successive dehydrogenation of the cyclohexyl radical is important to benzene formation close to the burner surface.

In this work, both 1-hexene and the cyclohexyl radical are formed in the pyrolysis of cyclohexane. ROP analysis of benzene in Fig. 8a was carried out at $1520 \mathrm{~K}$ to elucidate the benzene formation channels. As seen from Fig. 8a that benzene is formed by multiple channels, $32 \%$ benzene is formed from dehydrogenation of cyclohexadienyl (R39), 21\% benzene is formed from the isomerization of fulvene, which is formed by $\mathrm{C} 3+\mathrm{C} 3$ channels, i.e. $\mathrm{C}_{3} \mathrm{H}_{3}+\mathrm{aC}_{3} \mathrm{H}_{5}(67 \%)$ and $\mathrm{C}_{3} \mathrm{H}_{3}+\mathrm{C}_{3} \mathrm{H}_{3}(31 \%)$, and the rest is directly produced by combination of resonantly stabilized small radicals like $\mathrm{C}_{3} \mathrm{H}_{3}+\mathrm{C}_{3} \mathrm{H}_{3}, \mathrm{C}_{3} \mathrm{H}_{3}+\mathrm{aC}_{3} \mathrm{H}_{4}$ and $\mathrm{C}_{3} \mathrm{H}_{3}+\mathrm{pC}_{3} \mathrm{H}_{4}$. For the combination channels of benzene formation, the recombination of $\mathrm{C}_{3} \mathrm{H}_{3}$ is dominant (23\%). Considering the $\mathrm{C}_{3} \mathrm{H}_{3}$-based fulvene route and other reactions, $\mathrm{C} 3+\mathrm{C} 3$ reactions totally contribute about $58 \%$ to benzene formation. As seen in Fig. $3 \mathrm{f}$, the model predicts the measured mole fraction of benzene within the experimental uncertainty. The model simulates well the initial formation and peak temperature of fulvene, but the maximum mole fraction is overestimated. A larger uncertainty is associated with the measured mole fraction of fulvene since its PICS is unknown. Fig. 9 gives the sensitivity analysis of benzene performed at $1520 \mathrm{~K}$. It can be seen that the reaction of $\mathrm{CC}_{6} \mathrm{H}_{12}=\mathrm{C}_{6} \mathrm{H}_{12}$ (R1) has large positive coefficient. This confirms that the $\mathrm{C} 3+\mathrm{C} 3$ reactions play an important role for benzene formation because most $\mathrm{C} 3$ products are formed from 1-hexene. Stepwise dehydrogenation of cyclohexane via 


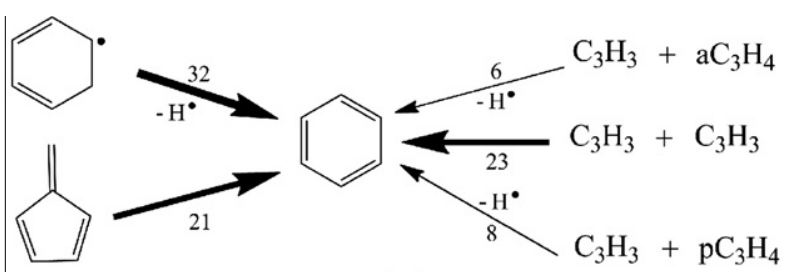

(a)
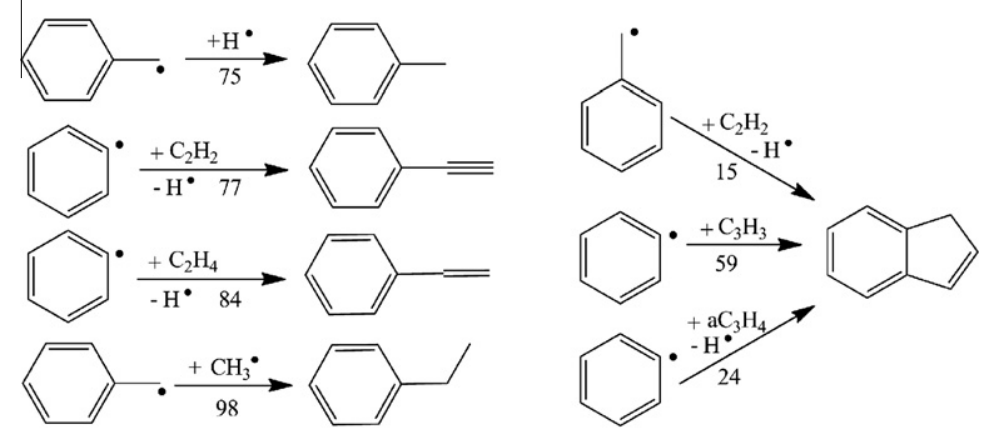

(b)

Fig. 8. ROP analysis of (a) benzene and (b) larger aromatics at $1520 \mathrm{~K}$. The numbers indicate the conversion percentage.

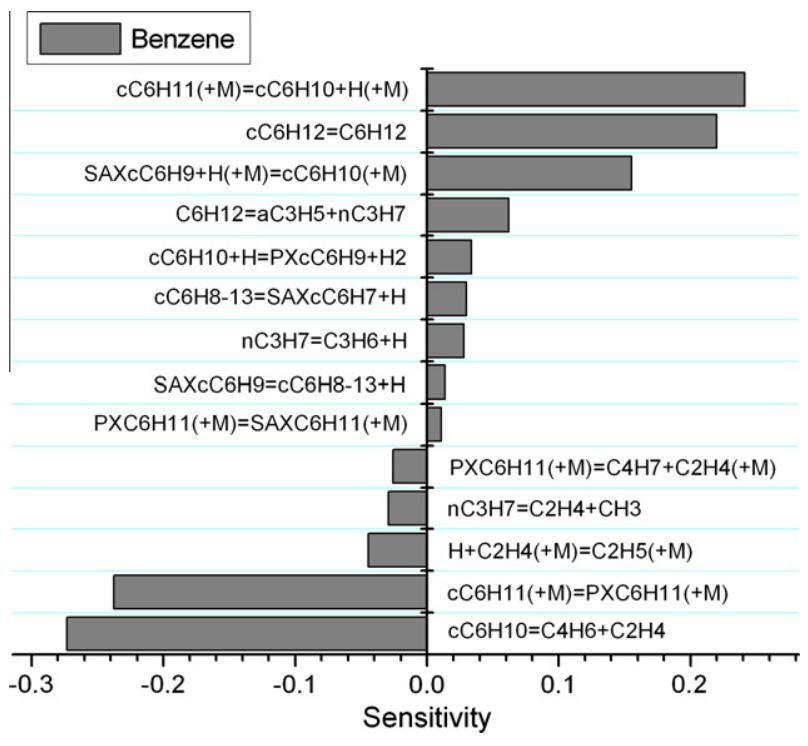

Fig. 9. Sensitivity analysis of benzene at $1520 \mathrm{~K}$. Reactions with absolute sensitivity coefficients larger than 0.01 are considered.

$\mathrm{cC}_{6} \mathrm{H}_{11} \rightarrow \mathrm{cC}_{6} \mathrm{H}_{10} \rightarrow \mathrm{SAXcC}_{6} \mathrm{H}_{9} \rightarrow \mathrm{cC}_{6} \mathrm{H}_{8}-13 \rightarrow$ cyclohexadienyl also has large positive coefficients for benzene formation.

Besides benzene, other aromatics were also detected, as shown in Fig. $3 \mathrm{~g}$ and $\mathrm{h}$. ROP analysis was performed at $1520 \mathrm{~K}$ to reveal the formation channels of these products. It can be seen from Fig. $8 \mathrm{~b}$ that these aromatics are mainly formed by addition reactions, i.e. benzyl $+\mathrm{H}$, phenyl $+\mathrm{C}_{2} \mathrm{H}_{2}$, phenyl $+\mathrm{C}_{2} \mathrm{H}_{4}$, benzyl $+\mathrm{CH}_{3}$, benzyl $+\mathrm{C}_{2} \mathrm{H}_{2}$, phenyl $+\mathrm{C}_{3} \mathrm{H}_{3}$ and phenyl + allene. Benzyl is dominantly formed by reaction benzyl $+\mathrm{H}=$ phenyl $+\mathrm{CH}_{3}$, and about $90 \%$ of the phenyl radical is formed by reaction $\mathrm{C}_{3} \mathrm{H}_{3}+\mathrm{C}_{3} \mathrm{H}_{3}=$ phenyl $+\mathrm{H}$. Therefore $\mathrm{C}_{3} \mathrm{H}_{3}+\mathrm{C}_{3} \mathrm{H}_{3}$ reaction plays an important role in larger aromatics formation.

\section{Conclusions}

The low-pressure pyrolysis of cyclohexane was studied in a plug flow reactor from 950 to $1520 \mathrm{~K}$ by using synchrotron VUV photoionization mass spectrometry. More than 30 species were identified and quantified. Isomerization to 1-hexene is found to be the dominant initial decomposition channel of cyclohexane pyrolysis under the low pressure condition. A kinetic model containing 148 species and 557 reactions was constructed to simulate the experimental results, and the simulated results agree well with experimental data for most of pyrolysis products. The ROP analysis under 1360 and $1520 \mathrm{~K}$ shows that cyclohexane is consumed mainly through the reaction sequence of cyclohexane $\rightarrow 1$-hexene $\rightarrow$ allyl radical $+n$-propyl radical and cyclohexane $\rightarrow$ cyclohexyl radical $\rightarrow$ hex-5-en-1-yl radical, which further decomposes to 1,3-butadiene via the hex-1-en-3-yl and but-3en-1-yl radicals. Multiple channels were found for benzene formation, i.e. combination of resonantly stabilized radicals and stepwise dehydrogenation of cyclohexane. Besides benzene, some aromatics i.e. toluene, phenylacetylene, styrene, ethylbenzene and indene were detected and quantified. The simulation reveals that $\mathrm{C}_{3} \mathrm{H}_{3}+$ $\mathrm{C}_{3} \mathrm{H}_{3}=$ phenyl $+\mathrm{H}$ is the key step for the formation of these larger aromatics.

\section{Acknowledgments}

Authors are grateful for the reviewers' insightful suggestions and the funding support from National Basic Research Program of China (973 Program) (2012CB719701), Natural Science Foundation of China (10979067 and 50925623), and Chinese Academy of Sciences.

\section{Appendix A. Supplementary material}

Supplementary data associated with this article can be found, in the online version, at doi:10.1016/j.combustflame.2012.02.019.

\section{References}

[1] C.K. Westbrook, Global Climate and Energy Project; Stanford University, June 13-16, 2005. <http://gcep.stanford.edu/pdfs/uQx8GXJG882-3q6NMuyQOw/ westbrook_symp05.pdf $>$. 
[2] J. Guthrie, P. Fowler, R. Sabourin, Gasoline and Diesel Fuel Survey, Environment Canada: Gatineau, Quebec, Canada, 2003. http://www.ec.gc.ca/cleanair-airpur/ CAOL/OGEB/fuels/reports/fullreport/pdf/FullReport2003-e.pdf.

[3] F. Billaud, M. Duret, K. Elyahyaoui, F. Baronnet, Ind. Eng. Chem. Res. 30 (1991) $1469-1478$.

[4] W.-C. Lai, C.S. Song, Fuel Process. Technol. 48 (1996) 1-27.

[5] J.H. Kiefer, K.S. Gupte, L.B. Harding, S.J. Klippenstein, J. Phys. Chem. A 113 (2009) 13570-13583.

[6] S. Peukert, C. Naumann, M. Braun-Unkhoff, U. Riedel, Int. J. Chem. Kinet. 43 (2011) 107-119.

[7] S.K. Gulati, R.W. Walker, J. Chem. Soc. Faraday Trans. 2 (85) (1989) 1799-1812.

[8] D. Voisin, A. Marchal, M. Reuillon, J.C. Boettner, M. Cathonnet, Combust. Sci. Technol. 138 (1998) 137-158.

[9] A. El Bakali, M. Braun-Unkhoff, P. Dagaut, P. Frank, M. Cathonnet, Proc. Combust. Inst. 28 (2000) 1631-1638.

[10] O. Lemaire, M. Ribaucour, M. Carlier, R. Minetti, Combust. Flame 127 (2001) 1971-1980.

[11] S. Granata, T. Faravelli, E. Ranzi, Combust. Flame 132 (2003) 533-544.

[12] F. Buda, B. Heyberger, R. Fournet, P.A. Glaude, V. Warth, F. Battin-Leclerc, Energy Fuels 20 (2006) 1450-1459.

[13] E.J. Silke, W.J. Pitz, C.K. Westbrook, M. Ribaucour, J. Phys. Chem. A 111 (2007) 3761-3775.

[14] B. Sirjean, F. Buda, H. Hakka, P.A. Glaude, R. Fournet, V. Warth, F. Battin-Leclerc, M. Ruiz-Lopez, Proc. Combust. Inst. 31 (2007) 277-284.

[15] S.M. Daley, A.M. Berkowitz, M.A. Oehlschlaeger, Int. J. Chem. Kinet. 40 (2008) 624-634.

[16] T. Ziehn, K.J. Hughes, J.F. Griffiths, R. Porter, A.S. Tomlin, Combust. Theor Model. 13 (2009) 589-605.

[17] Y. Yang, A.L. Boehman, Proc. Combust. Inst. 32 (2009) 419-426.

[18] P.J. Bennett, D. Gregory, R.A. Jackson, Combust. Sci. Technol. 115 (1996) 83103.

[19] S.G. Davis, C.K. Law, Combust. Sci. Technol. 140 (1998) 427-449.

[20] C.S. McEnally, L.D. Pfefferle, Combust. Flame 136 (2004) 155-167.

[21] M.E. Law, P.R. Westmoreland, T.A. Cool, J. Wang, N. Hansen, C.A. Taatjes, T. Kasper, Proc. Combust. Inst. 31 (2007) 565-573.

[22] H.Z.R. Zhang, L.K. Huynh, N. Kungwan, Z.W. Yang, S.W. Zhang, J. Phys. Chem. A 111 (2007) 4102-4115.

[23] A. Ciajolo, A. Tregrossi, M. Mallardo, T. Faravelli, E. Ranzi, Proc. Combust. Inst. 32 (2009) 585-591.

[24] C.S. Ji, E. Dames, B. Sirjean, H. Wang, F.N. Egolfopoulos, Proc. Combust. Inst. 33 (2011) 971-978.

[25] W.J. Li, M.E. Law, P.R. Westmoreland, T. Kasper, N. Hansen, K. KohseHöinghaus, Combust. Flame 158 (2011) 2077-2089.

[26] S.M. Handford-Styring, R.W. Walker, Phys. Chem. Chem. Phys. 3 (2001) 20432052.

[27] B. Sirjean, P.A. Glaude, M.F. Ruiz-Lopez, R. Fournet, J. Phys. Chem. A 110 (2006) 12693-12704

[28] C. Cavallotti, R. Rota, T. Faravelli, E. Ranzi, Proc. Combust. Inst. 31 (2007) 201209.

[29] W. Tsang, Int. J. Chem. Kinet. 10 (1978) 1119-1138.

[30] T.C. Brown, K.D. King, T.T. Nguyent, J. Phys. Chem. 90 (1986) 419-424.

[31] D.S. Aribike, A.A. Susu, A.F. Ogunye, Thermochim. Acta 51 (1981) 113-127.

[32] F. Billaud, P. Chaverot, M. Berthelin, E. Freund, Ind. Eng. Chem. Res. 27 (1988) 759-764.

[33] N. Hansen, T.A. Cool, P.R. Westmoreland, K. Kohse-Höinghaus, Prog. Energy Combust. Sci. 35 (2009) 168-191.

[34] Y.Y. Li, F. Qi, Acc. Chem. Res. 43 (2010) 68-78.

[35] Y. Pan, L.D. Zhang, H.J. Guo, L.L. Deng, F. Qi, Int. Rev. Phys. Chem. 29 (2010) 369-401.

[36] F. Battin-Leclerc, O. Herbinet, P.-A. Glaude, R. Fournet, Z.Y. Zhou, L.L. Deng, H.J. Guo, M.F. Xie, F. Qi, Angew. Chem. Int. Ed. 49 (2010) 3169-3172.

[37] Z.Y. Zhou, H.J. Guo, F. Qi, TrAC Trends Anal. Chem. 30 (2011) 1400-1409.

[38] F. Qi, R. Yang, B. Yang, C.Q. Huang, L.X. Wei, J. Wang, L.S. Sheng, Y.W. Zhang, Rev. Sci. Instrum. 77 (2006) 084101.

[39] T.C. Zhang, J. Wang, T. Yuan, X. Hong, L.D. Zhang, F. Qi, J. Phys. Chem. A 112 (2008) 10487-10494.

[40] Y.J. Zhang, J.H. Cai, L. Zhao, J.Z. Yang, H.F. Jin, Z.J. Cheng, Y.Y. Li, L.D. Zhang, F. Qi, Combust. Flame 159 (2012) 905-917.

[41] CHEMKIN-PRO 15092, Reaction Design, San Diego, 2009

[42] H. Wang, E. Dames, B. Sirjean, D.A. Sheen, R. Tangko, A. Violi, J.Y.W. Lai, F.N. Egolfopoulos, D.F. Davidson, R.K. Hanson, C.T. Bowman, C.K. Law, W. Tsang,
N.P. Cernansky, D.L. Miller, R.P. Lindstedt, A High-temperature Chemical Kinetic Model of n-alkane (up to n-dodecane), Cyclohexane, and Methyl-, ethyl-, n-propyl and n-butyl-cyclohexane Oxidation at High Temperatures, JetSurF Version 2.0, September 19, 2010. <http://melchior.usc.edu/JetSurF/ JetSurF2.0>.

[43] E. Goos, A. Burcat, B. Ruscic, Ideal Gas Thermochemical Database with updates from Active Thermochemical Tables. <ftp://ftp.technion.ac.il/pub/supported/ aetdd/thermodynamics>.

[44] J.H. Kiefer, J.N. Shah, J. Phys. Chem. 91 (1987) 3024-3030.

[45] L.D. Zhang, J.H. Cai, T.C. Zhang, F. Qi, Combust. Flame 157 (2010) 1686-1697.

[46] Y.Y. Li, J.H. Cai, L.D. Zhang, T. Yuan, K.W. Zhang, F. Qi, Proc. Combust. Inst. 33 (2011) 593-600.

[47] Y.Y. Li, J.H. Cai, L.D. Zhang, J.Z. Yang, Z.D. Wang, F. Qi, Proc. Combust. Inst. 33 (2011) 617-624.

[48] J.A. Miller, S.J. Klippenstein, J. Phys. Chem. A 107 (2003) 7783-7799.

[49] Y. Georgievskii, J.A. Miller, S.J. Klippenstein, Phys. Chem. Chem. Phys. 9 (2007) 4259-4268.

[50] S. Scherer, T. Just, P. Frank, Proc. Combust. Inst. 28 (2000) 1511-1518.

[51] R.X. Fernandes, H. Hippler, M. Olzmann, Proc. Combust. Inst. 30 (2005) 10331038.

[52] K. Roy, C. Horn, P. Frank, V.G. Slutsky, T. Just, Proc. Combust. Inst. 27 (1998) 329-336.

[53] G.B. Bacskay, J.C. Mackie, Phys. Chem. Chem. Phys. 3 (2001) 2467-2473.

[54] H. Wang, X. You, A.V. Joshi, S.G. Davis, A. Laskin, F. Egolfopoulos, C.K. Law, High-Temperature Combustion Reaction Model of $\mathrm{H}_{2} / \mathrm{CO} / \mathrm{C} 1-\mathrm{C} 4$ Compounds, 2007. http://ignis.usc.edu/USC_Mech_II.htm.

[55] H. Richter, S. Granata, W.H. Green, J.B. Howard, Proc. Combust. Inst. 30 (2005) 1397-1405.

[56] A. Laskin, H. Wang, C.K. Law, Int. J. Chem. Kinet. 32 (2000) 589-614.

[57] J.A. Miller, J.P. Senosiain, S.J. Klippenstein, Y. Georgievskii, J. Phys. Chem. A 112 (2008) 9429-9438.

[58] T. Bentz, B.R. Giri, H. Hippler, M. Olzmann, F. Striebel, M. Szöri, J. Phys. Chem. A 111 (2007) 3812-3818

[59] N. Hansen, T. Kasper, B. Yang, T.A. Cool, W.J. Li, P.R. Westmoreland, P. Oßwald, K. Kohse-Höinghaus, Proc. Combust. Inst. 33 (2011) 585-592.

[60] N. Hansen, W.J. Li, M.E. Law, T. Kasper, P.R. Westmoreland, B. Yang, T.A. Cool, A. Lucassen, Phys. Chem. Chem. Phys. 12 (2010) 12112-12122.

[61] G. Dayma, P.A. Glaude, R. Fournet, F. Battin-Leclerc, Int. J. Chem. Kinet. 35 (2003) 273-285.

[62] A. D’Anna, A. D’Alessio, J. Kent, Combust. Flame 125 (2001) 1196-1206.

[63] N. Hansen, J.A. Miller, P.R. Westmoreland, T. Kasper, K. Kohse-Höinghaus, J. Wang, T.A. Cool, Combust. Flame 156 (2009) 2153-2164.

[64] R. Sivaramakrishnan, R.S. Tranter, K. Brezinsky, J. Phys. Chem. A 110 (2006) 9388-9399.

[65] R.P. Lindstedt, G. Skevis, Combust. Sci. Technol. 125 (1997) 73-137.

[66] J.P. Senosiain, J.A. Miller, J. Phys. Chem. A 111 (2007) 3740-3747.

[67] C.A. Taatjes, D.L. Osborn, T.M. Selby, G. Meloni, H.Y. Fan, S.T. Pratt, J. Phys. Chem. A 112 (2008) 9336-9343.

[68] T.A. Cool, J. Wang, K. Nakajima, C.A. Taatjes, A. McLlroy, Int. J. Mass Spectrom. 247 (2005) 18-27.

[69] J.C. Robinson, N.E. Sveum, D.M. Neumark, J. Chem. Phys. 119 (2003) 53115314.

[70] B. Yang, J. Wang, T.A. Cool, N. Hansen, S. Skeen, D.L. Osborn, Int. J. Mass Spectrom. 309 (2012) 118-128.

[71] J.C. Robinson, N.E. Sveum, D.M. Neumark, Chem. Phys. Lett. 383 (2004) 601605.

[72] T.A. Cool, K. Nakajima, T.A. Mostefaoui, F. Qi, A. Mcllroy, P.R. Westmoreland, M.E. Law, L. Poisson, D.S. Peterka, M. Ahmed, J. Chem. Phys. 119 (2003) 83568365.

[73] H. Koizumi, J. Chem. Phys. 95 (1991) 5846-5852.

[74] J. Wang, B. Yang, T.A. Cool, N. Hansen, T. Kasper, Int. J. Mass Spectrom. 269 (2008) 210-220.

[75] N. Hansen, S.J. Klippenstein, J.A. Miller, J. Wang, T.A. Cool, M.E. Law, P.R. Westmoreland, T. Kasper, K. Kohse-Höinghaus, J. Phys. Chem. A 110 (2006) $4376-4388$.

[76] Z.Y. Zhou, L.D. Zhang, M.F. Xie, Z.D. Wang, D.N. Chen, F. Qi, Rapid Commun. Mass Spectrom. 24 (2010) 1335-1342.

[77] Z.Y. Zhou, M.F. Xie, Z.D. Wang, F. Qi, Rapid Commun. Mass Spectrom. 23 (2009) 3994-4002. 